\title{
Synthesis and Photophysical Properties of Trimetallic Acetylide Complexes with a
}

\section{1,3,5-Triazine Core}

Quan Yuan Hu, ${ }^{a}$ Wei Xin Lu, ${ }^{\mathrm{b}}$ Hong Ding Tang, ${ }^{\mathrm{a}}$ Herman H. Y. Sung, ${ }^{\mathrm{a}}$ Ting Bin Wen, ${ }^{a}$ Ian D. Williams, ${ }^{a}$ George K. L. Wong, ${ }^{b} *$ Zhenyang $\operatorname{Lin}^{\mathrm{a}, *}$ and Guochen Jia ${ }^{\mathrm{a}, *}$ aDepartment of Chemistry and Open Laboratory of Chirotechnology of the Institute of Molecular Technology for Drug Discovery and Synthesis and bepartment of Physics, The Hong Kong University of Science and Technology, Clear Water Bay, Kowloon, Hong Kong

\section{Supporting Information Available}

Table S1. Atomic coordinates $\left(\times 10^{4}\right)$ and equivalent isotropic displacement parameters $\left(\AA^{2} \times 10^{3}\right)$ for $2,4,6-\left[4-\left(\mathrm{Cl}(\mathrm{dppe})_{2} \mathrm{RuC} \equiv \mathrm{C}\right) \mathrm{C}_{6} \mathrm{H}_{4}\right]_{3}-1,3,5-\mathrm{C}_{3} \mathrm{~N}_{3}(\mathbf{5})$. U(eq) is defined as one third of the trace of the orthogonalized Uij tensor

Table S2. Bond Lengths $[\AA]$ and angles $\left[^{\circ}\right]$ for $2,4,6-\left[4-\left(\mathrm{Cl}(\text { dppe })_{2} \mathrm{RuC} \equiv \mathrm{C}\right) \mathrm{C}_{6} \mathrm{H}_{4}\right]_{3^{-}}$ $1,3,5-\mathrm{C}_{3} \mathrm{~N}_{3}(\mathbf{5})$

Table S3. Anisotropic displacement parameters $\left(\AA^{2} \times 10^{3}\right)$ for 2,4,6-[4$\left.\left(\mathrm{Cl}(\mathrm{dppe}){ }_{2} \mathrm{RuC} \equiv \mathrm{C}\right) \mathrm{C}_{6} \mathrm{H}_{4}\right]_{3}-1,3,5-\mathrm{C}_{3} \mathrm{~N}_{3}(\mathbf{5})$

Table S4. Hydrogen coordinates $\left(\times 10^{4}\right)$ and isotropic displacement parameters $\left(\AA^{2} \times 10^{3}\right)$ for $2,4,6-\left[4-\left(\mathrm{Cl}(\mathrm{dppe}){ }_{2} \mathrm{RuC} \equiv \mathrm{C}\right) \mathrm{C}_{6} \mathrm{H}_{4}\right]_{3}-1,3,5-\mathrm{C}_{3} \mathrm{~N}_{3}(\mathbf{5})$ 
Table S1. Atomic coordinates ( $\left.10^{4}\right)$ and equivalent isotropic displacement parameters $\left(\AA^{2} \times 10^{3}\right)$ for 2,4,6-[4- $\left.\left(\mathrm{Cl}(\mathrm{dppe})_{2} \mathrm{RuC} \equiv \mathrm{C}\right) \mathrm{C}_{6} \mathrm{H}_{4}\right]_{3}-1,3,5-\mathrm{C}_{3} \mathrm{~N}_{3}(\mathbf{5})$. U(eq) is defined as one third of the trace of the orthogonalized $\mathrm{U}^{\mathrm{ij}}$ tensor.

\begin{tabular}{|c|c|c|c|c|}
\hline & $\mathrm{x}$ & $\mathrm{y}$ & $\mathrm{z}$ & $\mathrm{U}(\mathrm{eq})$ \\
\hline $\operatorname{Ru}(1)$ & $6727(1)$ & $5069(1)$ & 1933(1) & $21(1)$ \\
\hline $\operatorname{Ru}(2)$ & $-1117(1)$ & $-966(1)$ & $1434(1)$ & $21(1)$ \\
\hline $\operatorname{Ru}(3)$ & $7240(1)$ & $-2574(1)$ & $5377(1)$ & $18(1)$ \\
\hline $\mathrm{Cl}(1)$ & $7469(1)$ & 6134(1) & $1822(1)$ & $29(1)$ \\
\hline $\mathrm{Cl}(2)$ & $-2459(1)$ & $-1374(1)$ & $1075(1)$ & $30(1)$ \\
\hline $\mathrm{Cl}(3)$ & $7915(1)$ & $-3338(1)$ & $6009(1)$ & $24(1)$ \\
\hline $\mathrm{P}(11)$ & $7520(1)$ & $4500(1)$ & $1439(1)$ & $26(1)$ \\
\hline $\mathrm{P}(12)$ & $5923(1)$ & $5154(1)$ & $1145(1)$ & $26(1)$ \\
\hline $\mathrm{P}(13)$ & $5905(1)$ & $5605(1)$ & 2391(1) & $24(1)$ \\
\hline $\mathrm{P}(14)$ & $7496(1)$ & $5009(1)$ & $2725(1)$ & $26(1)$ \\
\hline $\mathrm{P}(21)$ & $-848(1)$ & $-835(1)$ & $656(1)$ & $25(1)$ \\
\hline $\mathrm{P}(22)$ & $-1494(1)$ & $240(1)$ & $1410(1)$ & $25(1)$ \\
\hline $\mathrm{P}(23)$ & $-1395(1)$ & $-1094(1)$ & $2205(1)$ & $24(1)$ \\
\hline $\mathrm{P}(24)$ & $-775(1)$ & $-2183(1)$ & $1503(1)$ & $23(1)$ \\
\hline $\mathrm{P}(31)$ & $8051(1)$ & $-2683(1)$ & $4822(1)$ & $22(1)$ \\
\hline $\mathrm{P}(32)$ & $6510(1)$ & $-3462(1)$ & $4777(1)$ & $20(1)$ \\
\hline $\mathrm{P}(33)$ & 6392(1) & $-2449(1)$ & $5900(1)$ & $19(1)$ \\
\hline $\mathrm{P}(34)$ & $7868(1)$ & $-1571(1)$ & $5928(1)$ & $21(1)$ \\
\hline $\mathrm{N}(1)$ & $5104(1)$ & $808(1)$ & $3129(1)$ & $18(1)$ \\
\hline $\mathrm{N}(2)$ & 3961(1) & 1009(1) & $2564(1)$ & $22(1)$ \\
\hline $\mathrm{N}(3)$ & $4015(1)$ & $49(1)$ & $3052(1)$ & $20(1)$ \\
\hline $\mathrm{C}(11)$ & $4690(1)$ & 1163(1) & $2783(1)$ & $18(1)$ \\
\hline$C(12)$ & $5059(1)$ & $1785(1)$ & $2623(1)$ & $21(1)$ \\
\hline $\mathrm{C}(13)$ & $5731(1)$ & $2115(1)$ & $2900(1)$ & $21(1)$ \\
\hline$C(14)$ & $6034(2)$ & $2708(1)$ & $2753(1)$ & $25(1)$ \\
\hline$C(15)$ & $5675(1)$ & 2997(1) & $2319(1)$ & $20(1)$ \\
\hline$C(16)$ & $5002(2)$ & $2668(1)$ & $2049(1)$ & $28(1)$ \\
\hline $\mathrm{C}(17)$ & $4691(2)$ & 2073(1) & 2193(1) & $27(1)$ \\
\hline $\mathrm{C}(18)$ & $5990(2)$ & $3630(1)$ & $2167(1)$ & $24(1)$ \\
\hline$C(19)$ & $6228(2)$ & $4160(1)$ & $2046(1)$ & $23(1)$ \\
\hline $\mathrm{C}(21)$ & $3654(1)$ & $449(1)$ & $2706(1)$ & $20(1)$ \\
\hline$C(22)$ & $2855(1)$ & $250(1)$ & $2474(1)$ & $21(1)$ \\
\hline
\end{tabular}




\begin{tabular}{|c|c|c|c|c|}
\hline $\mathrm{C}(23)$ & $2398(2)$ & $731(2)$ & $2218(1)$ & $27(1)$ \\
\hline $\mathrm{C}(24)$ & $1643(2)$ & $554(2)$ & $2018(1)$ & $29(1)$ \\
\hline$C(25)$ & $1342(1)$ & $-127(2)$ & $2053(1)$ & $24(1)$ \\
\hline$C(26)$ & 1811(2) & $-614(1)$ & $2303(1)$ & $24(1)$ \\
\hline $\mathrm{C}(27)$ & $2552(1)$ & $-424(1)$ & $2513(1)$ & $22(1)$ \\
\hline $\mathrm{C}(28)$ & $562(2)$ & $-350(1)$ & 1831(1) & $25(1)$ \\
\hline $\mathrm{C}(29)$ & $-67(1)$ & $-580(1)$ & 1693(1) & $21(1)$ \\
\hline $\mathrm{C}(31)$ & $4735(1)$ & $254(1)$ & $3258(1)$ & $18(1)$ \\
\hline$C(32)$ & $5146(1)$ & $-176(1)$ & $3627(1)$ & $21(1)$ \\
\hline $\mathrm{C}(33)$ & $4772(2)$ & $-713(1)$ & $3806(1)$ & $26(1)$ \\
\hline$C(34)$ & $5136(2)$ & $-1128(1)$ & $4153(1)$ & $22(1)$ \\
\hline$C(35)$ & $5915(2)$ & $-1030(1)$ & $4337(1)$ & $23(1)$ \\
\hline$C(36)$ & $6296(1)$ & $-474(1)$ & $4174(1)$ & $20(1)$ \\
\hline $\mathrm{C}(37)$ & $5912(2)$ & $-57(1)$ & $3828(1)$ & $24(1)$ \\
\hline $\mathrm{C}(38)$ & $6330(1)$ & $-1493(1)$ & $4676(1)$ & $21(1)$ \\
\hline$C(39)$ & $6671(1)$ & $-1902(1)$ & 4941(1) & $19(1)$ \\
\hline $\mathrm{C}(101)$ & $7206(2)$ & $4832(2)$ & $838(1)$ & $31(1)$ \\
\hline C(102) & $6353(2)$ & $4700(2)$ & $666(1)$ & $32(1)$ \\
\hline $\mathrm{C}(103)$ & $6117(2)$ & $5198(1)$ & $2975(1)$ & $26(1)$ \\
\hline $\mathrm{C}(104)$ & $6955(2)$ & 5303(2) & 3191(1) & $27(1)$ \\
\hline $\mathrm{C}(111)$ & $8541(2)$ & $4679(2)$ & 1594(1) & $29(1)$ \\
\hline $\mathrm{C}(112)$ & $8862(2)$ & $5235(2)$ & $1403(1)$ & $41(1)$ \\
\hline $\mathrm{C}(113)$ & $9650(2)$ & $5321(2)$ & $1508(1)$ & $53(1)$ \\
\hline $\mathrm{C}(114)$ & $10103(2)$ & $4890(2)$ & $1803(1)$ & $53(1)$ \\
\hline$C(115)$ & $9793(2)$ & $4332(2)$ & 1988(1) & $45(1)$ \\
\hline $\mathrm{C}(116)$ & $9008(2)$ & $4247(2)$ & 1884(1) & $38(1)$ \\
\hline $\mathrm{C}(121)$ & $7460(2)$ & $3521(2)$ & 1207(1) & $28(1)$ \\
\hline $\mathrm{C}(122)$ & $6842(2)$ & $3094(2)$ & $1214(1)$ & $36(1)$ \\
\hline $\mathrm{C}(123)$ & $6737(2)$ & $2382(2)$ & $974(1)$ & $42(1)$ \\
\hline C(124) & $7266(2)$ & 2093(2) & 724(1) & $44(1)$ \\
\hline$C(125)$ & $7887(2)$ & $2515(2)$ & $725(1)$ & $39(1)$ \\
\hline$C(126)$ & $7987(2)$ & $3223(2)$ & $957(1)$ & $37(1)$ \\
\hline $\mathrm{C}(131)$ & $5763(2)$ & $6060(2)$ & $956(1)$ & $33(1)$ \\
\hline $\mathrm{C}(132)$ & $5602(2)$ & $6180(2)$ & $475(1)$ & $63(1)$ \\
\hline C(133) & $5511(3)$ & $6873(2)$ & 351(1) & $98(2)$ \\
\hline $\mathrm{C}(134)$ & $5626(2)$ & $7464(2)$ & $725(2)$ & $85(2)$ \\
\hline$C(135)$ & $5754(2)$ & $7349(2)$ & $1210(1)$ & $63(1)$ \\
\hline $\mathrm{C}(136)$ & $5835(2)$ & $6648(2)$ & 1319(1) & $43(1)$ \\
\hline
\end{tabular}




\begin{tabular}{|c|c|c|c|c|}
\hline $\mathrm{C}(141)$ & $4976(2)$ & $4714(2)$ & $970(1)$ & $37(1)$ \\
\hline $\mathrm{C}(142)$ & $4886(2)$ & $3975(2)$ & 1001(1) & $41(1)$ \\
\hline C(143) & $4186(2)$ & $3624(2)$ & $874(1)$ & $54(1)$ \\
\hline C(144) & $3574(2)$ & $4013(2)$ & $721(1)$ & $62(1)$ \\
\hline$C(145)$ & $3640(2)$ & $4749(2)$ & $694(1)$ & $53(1)$ \\
\hline $\mathrm{C}(146)$ & $4334(2)$ & $5113(2)$ & $819(1)$ & $46(1)$ \\
\hline $\mathrm{C}(151)$ & $5962(2)$ & $6564(1)$ & $2659(1)$ & $26(1)$ \\
\hline $\mathrm{C}(152)$ & $6502(2)$ & $7053(2)$ & $2597(1)$ & $33(1)$ \\
\hline$C(153)$ & $6540(2)$ & $7775(2)$ & $2829(1)$ & $41(1)$ \\
\hline $\mathrm{C}(154)$ & $6036(2)$ & $8010(2)$ & $3104(1)$ & $41(1)$ \\
\hline$C(155)$ & $5485(2)$ & $7523(1)$ & $3164(1)$ & $34(1)$ \\
\hline$C(156)$ & $5455(2)$ & $6816(2)$ & $2954(1)$ & $28(1)$ \\
\hline $\mathrm{C}(161)$ & $4890(2)$ & $5461(1)$ & $2165(1)$ & $25(1)$ \\
\hline$C(162)$ & $4436(2)$ & $5998(2)$ & 1984(1) & $38(1)$ \\
\hline$C(163)$ & $3673(2)$ & $5896(2)$ & $1835(1)$ & $48(1)$ \\
\hline $\mathrm{C}(164)$ & $3326(2)$ & $5229(2)$ & $1856(1)$ & $49(1)$ \\
\hline$C(165)$ & $3764(2)$ & $4683(2)$ & $2023(1)$ & $38(1)$ \\
\hline $\mathrm{C}(166)$ & $4531(2)$ & $4799(2)$ & $2175(1)$ & $34(1)$ \\
\hline$C(171)$ & $7819(2)$ & $4133(2)$ & 2902(1) & $32(1)$ \\
\hline $\mathrm{C}(172)$ & $8102(2)$ & $4043(2)$ & $3401(1)$ & $49(1)$ \\
\hline$C(173)$ & $8321(2)$ & $3371(2)$ & $3532(1)$ & $62(1)$ \\
\hline$C(174)$ & $8276(2)$ & $2796(2)$ & $3153(1)$ & $60(1)$ \\
\hline $\mathrm{C}(175)$ & $8029(2)$ & $2873(2)$ & $2671(1)$ & $42(1)$ \\
\hline$C(176)$ & $7785(1)$ & $3536(2)$ & $2546(1)$ & $28(1)$ \\
\hline $\mathrm{C}(181)$ & $8334(2)$ & $5600(2)$ & 2934(1) & $35(1)$ \\
\hline $\mathrm{C}(182)$ & $8272(2)$ & $6354(2)$ & $3050(1)$ & $50(1)$ \\
\hline$C(183)$ & $8873(2)$ & $6850(2)$ & $3166(1)$ & $56(1)$ \\
\hline C(184) & $9564(2)$ & $6597(2)$ & $3153(1)$ & $67(1)$ \\
\hline $\mathrm{C}(185)$ & $9674(2)$ & $5896(2)$ & $3026(2)$ & $77(2)$ \\
\hline $\mathrm{C}(186)$ & $9033(2)$ & $5370(2)$ & 2918(1) & $65(1)$ \\
\hline $\mathrm{C}(201)$ & $-801(2)$ & $148(1)$ & $632(1)$ & $28(1)$ \\
\hline$C(202)$ & $-1493(2)$ & $463(2)$ & $785(1)$ & $30(1)$ \\
\hline$C(203)$ & $-1761(2)$ & $-2030(1)$ & 2132(1) & $25(1)$ \\
\hline$C(204)$ & $-1168(1)$ & $-2518(1)$ & 1999(1) & $24(1)$ \\
\hline$C(211)$ & $40(2)$ & $-1107(2)$ & $517(1)$ & $33(1)$ \\
\hline $\mathrm{C}(212)$ & $81(2)$ & $-1766(2)$ & 213(1) & $41(1)$ \\
\hline$C(213)$ & $752(2)$ & $-1995(2)$ & $138(1)$ & $52(1)$ \\
\hline $\mathrm{C}(214)$ & $1415(2)$ & $-1590(2)$ & $362(1)$ & $57(1)$ \\
\hline
\end{tabular}




\begin{tabular}{|c|c|c|c|c|}
\hline$C(215)$ & $1390(2)$ & $-941(2)$ & $651(1)$ & $44(1)$ \\
\hline$C(216)$ & $718(2)$ & $-694(2)$ & $726(1)$ & $33(1)$ \\
\hline$C(221)$ & $-1506(2)$ & $-1178(2)$ & $66(1)$ & $32(1)$ \\
\hline$C(222)$ & $-1967(2)$ & $-1780(2)$ & $31(1)$ & $37(1)$ \\
\hline$C(223)$ & $-2441(2)$ & $-2050(2)$ & $-417(1)$ & $59(1)$ \\
\hline$C(224)$ & $-2462(2)$ & $-1717(2)$ & $-845(1)$ & $71(2)$ \\
\hline$C(225)$ & $-2012(2)$ & $-1132(2)$ & $-807(1)$ & $81(2)$ \\
\hline$C(226)$ & $-1529(2)$ & $-871(2)$ & $-357(1)$ & $70(1)$ \\
\hline$C(231)$ & $-2421(2)$ & $525(2)$ & 1481(1) & $33(1)$ \\
\hline$C(232)$ & $-3077(2)$ & $280(2)$ & $1116(1)$ & $36(1)$ \\
\hline$C(233)$ & $-3773(2)$ & $511(2)$ & $1163(1)$ & $44(1)$ \\
\hline$C(234)$ & $-3827(2)$ & $983(2)$ & $1569(1)$ & $52(1)$ \\
\hline$C(235)$ & $-3206(2)$ & $1229(2)$ & 1936(1) & $47(1)$ \\
\hline$C(236)$ & $-2497(2)$ & $1009(2)$ & 1892(1) & $37(1)$ \\
\hline $\mathrm{C}(241)$ & $-873(2)$ & $948(2)$ & $1825(1)$ & $29(1)$ \\
\hline$C(242)$ & $-448(2)$ & $811(2)$ & 2281(1) & $28(1)$ \\
\hline$C(243)$ & $1(2)$ & $1349(2)$ & 2601(1) & $36(1)$ \\
\hline$C(244)$ & $51(2)$ & $2023(2)$ & $2476(1)$ & $55(1)$ \\
\hline$C(245)$ & $-346(2)$ & 2198(2) & 2034(2) & $71(2)$ \\
\hline$C(246)$ & $-803(2)$ & $1652(2)$ & $1699(1)$ & $55(1)$ \\
\hline$C(251)$ & $-2103(2)$ & $-566(1)$ & 2441(1) & $27(1)$ \\
\hline$C(252)$ & $-2863(2)$ & $-726(2)$ & $2224(1)$ & $35(1)$ \\
\hline$C(253)$ & $-3409(2)$ & $-333(2)$ & 2414(1) & $39(1)$ \\
\hline$C(254)$ & $-3221(2)$ & $200(2)$ & $2803(1)$ & $43(1)$ \\
\hline$C(255)$ & $-2471(2)$ & $375(2)$ & $3011(1)$ & $36(1)$ \\
\hline$C(256)$ & $-1912(2)$ & $-10(2)$ & $2830(1)$ & $34(1)$ \\
\hline$C(261)$ & $-654(2)$ & $-1113(1)$ & $2775(1)$ & $24(1)$ \\
\hline$C(262)$ & $-837(2)$ & $-1262(2)$ & $3212(1)$ & $32(1)$ \\
\hline$C(263)$ & $-285(2)$ & $-1373(2)$ & $3622(1)$ & $35(1)$ \\
\hline$C(264)$ & $456(2)$ & $-1321(2)$ & $3616(1)$ & $35(1)$ \\
\hline$C(265)$ & $662(2)$ & $-1164(2)$ & $3188(1)$ & $36(1)$ \\
\hline$C(266)$ & $110(2)$ & $-1071(2)$ & $2768(1)$ & $33(1)$ \\
\hline $\mathrm{C}(271)$ & $-1155(2)$ & $-2866(1)$ & $968(1)$ & $25(1)$ \\
\hline$C(272)$ & $-1918(2)$ & $-3080(1)$ & $852(1)$ & $29(1)$ \\
\hline$C(273)$ & $-2229(2)$ & $-3564(2)$ & $436(1)$ & $32(1)$ \\
\hline$C(274)$ & $-1770(2)$ & $-3837(2)$ & $134(1)$ & $44(1)$ \\
\hline$C(275)$ & $-1013(2)$ & $-3647(2)$ & $242(1)$ & $49(1)$ \\
\hline$C(276)$ & $-706(2)$ & $-3153(2)$ & $662(1)$ & $33(1)$ \\
\hline
\end{tabular}




\begin{tabular}{|c|c|c|c|c|}
\hline $\mathrm{C}(281)$ & 202(2) & $-2437(2)$ & $1675(1)$ & $29(1)$ \\
\hline $\mathrm{C}(282)$ & $757(2)$ & $-2108(2)$ & $1482(1)$ & $32(1)$ \\
\hline$C(283)$ & $1487(2)$ & $-2329(2)$ & $1578(1)$ & $39(1)$ \\
\hline$C(284)$ & $1695(2)$ & $-2870(2)$ & 1871(1) & $43(1)$ \\
\hline$C(285)$ & $1153(2)$ & $-3190(2)$ & 2072(1) & $49(1)$ \\
\hline$C(286)$ & $432(2)$ & $-2989(2)$ & $1970(1)$ & $40(1)$ \\
\hline $\mathrm{C}(301)$ & $7537(1)$ & $-3231(2)$ & $4234(1)$ & $25(1)$ \\
\hline$C(302)$ & $6682(2)$ & $-3237(1)$ & $4182(1)$ & $24(1)$ \\
\hline$C(303)$ & $6822(1)$ & $-1798(1)$ & $6462(1)$ & $19(1)$ \\
\hline$C(304)$ & $7669(2)$ & $-1685(1)$ & $6531(1)$ & $26(1)$ \\
\hline$C(311)$ & $8948(2)$ & $-3150(1)$ & 4893(1) & $26(1)$ \\
\hline$C(312)$ & $9177(2)$ & $-3581(2)$ & $5267(1)$ & $34(1)$ \\
\hline$C(313)$ & $9822(2)$ & $-3976(2)$ & $5279(1)$ & $44(1)$ \\
\hline $\mathrm{C}(314)$ & $10216(2)$ & $-3945(2)$ & 4921(1) & $54(1)$ \\
\hline$C(315)$ & 9993(2) & $-3526(2)$ & $4546(1)$ & $54(1)$ \\
\hline$C(316)$ & $9359(2)$ & $-3130(2)$ & $4533(1)$ & $44(1)$ \\
\hline$C(321)$ & $8325(2)$ & $-1840(1)$ & $4620(1)$ & $26(1)$ \\
\hline$C(322)$ & $8982(2)$ & $-1451(2)$ & $4896(1)$ & $33(1)$ \\
\hline$C(323)$ & $9148(2)$ & $-758(2)$ & $4779(1)$ & $43(1)$ \\
\hline$C(324)$ & $8700(2)$ & $-485(2)$ & $4379(1)$ & $49(1)$ \\
\hline$C(325)$ & $8076(2)$ & $-877(2)$ & $4100(1)$ & $46(1)$ \\
\hline$C(326)$ & $7878(2)$ & $-1547(2)$ & $4211(1)$ & $32(1)$ \\
\hline$C(331)$ & $6704(2)$ & $-4441(1)$ & $4703(1)$ & $22(1)$ \\
\hline$C(332)$ & $6258(2)$ & $-4911(1)$ & $4308(1)$ & $25(1)$ \\
\hline$C(333)$ & $6438(2)$ & $-5624(2)$ & $4215(1)$ & $30(1)$ \\
\hline$C(334)$ & $7068(2)$ & $-5881(2)$ & $4514(1)$ & $36(1)$ \\
\hline$C(335)$ & $7506(2)$ & $-5427(2)$ & $4908(1)$ & $33(1)$ \\
\hline$C(336)$ & $7327(2)$ & $-4707(1)$ & 4999(1) & $29(1)$ \\
\hline$C(341)$ & $5481(1)$ & $-3503(1)$ & $4655(1)$ & 19(1) \\
\hline $\mathrm{C}(342)$ & $5097(2)$ & $-4004(1)$ & $4849(1)$ & $24(1)$ \\
\hline $\mathrm{C}(343)$ & $4326(2)$ & $-4020(1)$ & $4757(1)$ & $26(1)$ \\
\hline$C(344)$ & $3924(2)$ & $-3554(2)$ & $4474(1)$ & $30(1)$ \\
\hline$C(345)$ & $4297(2)$ & $-3046(2)$ & $4278(1)$ & $31(1)$ \\
\hline$C(346)$ & $5070(2)$ & $-3017(1)$ & $4379(1)$ & $23(1)$ \\
\hline$C(351)$ & $6119(1)$ & $-3236(1)$ & $6176(1)$ & $19(1)$ \\
\hline $\mathrm{C}(352)$ & $6264(1)$ & $-3932(1)$ & $6016(1)$ & $20(1)$ \\
\hline$C(353)$ & $6048(2)$ & $-4514(1)$ & $6229(1)$ & $25(1)$ \\
\hline $\mathrm{C}(354)$ & $5666(2)$ & $-4396(2)$ & $6600(1)$ & $31(1)$ \\
\hline
\end{tabular}




\begin{tabular}{|c|c|c|c|c|}
\hline$C(355)$ & $5510(2)$ & $-3706(2)$ & $6767(1)$ & $32(1)$ \\
\hline$C(356)$ & $5730(2)$ & $-3117(2)$ & $6554(1)$ & $28(1)$ \\
\hline$C(361)$ & $5490(1)$ & $-2054(1)$ & $5682(1)$ & $22(1)$ \\
\hline$C(362)$ & $4803(2)$ & $-2444(2)$ & $5604(1)$ & $29(1)$ \\
\hline C(363) & $4148(2)$ & $-2120(2)$ & $5435(1)$ & $36(1)$ \\
\hline$C(364)$ & $4127(2)$ & $-1428(2)$ & $5322(1)$ & $40(1)$ \\
\hline$C(365)$ & $4806(2)$ & $-1039(2)$ & $5385(1)$ & $38(1)$ \\
\hline$C(366)$ & $5471(2)$ & $-1343(1)$ & $5563(1)$ & $27(1)$ \\
\hline $\mathrm{C}(371)$ & $7588(1)$ & $-639(1)$ & $5832(1)$ & $22(1)$ \\
\hline $\mathrm{C}(372)$ & $7627(1)$ & $-389(1)$ & $5397(1)$ & $24(1)$ \\
\hline$C(373)$ & $7517(2)$ & $325(2)$ & $5330(1)$ & $31(1)$ \\
\hline C(374) & $7339(2)$ & $811(2)$ & $5706(1)$ & $33(1)$ \\
\hline$C(375)$ & $7299(2)$ & $566(2)$ & $6143(1)$ & $28(1)$ \\
\hline$C(376)$ & $7425(1)$ & $-164(1)$ & $6207(1)$ & $26(1)$ \\
\hline $\mathrm{C}(381)$ & $8892(1)$ & $-1435(1)$ & $6066(1)$ & $24(1)$ \\
\hline $\mathrm{C}(382)$ & $9256(2)$ & $-746(2)$ & $6132(1)$ & $25(1)$ \\
\hline C(383) & $10032(2)$ & $-677(2)$ & $6206(1)$ & $34(1)$ \\
\hline$C(384)$ & $10436(2)$ & $-1293(2)$ & $6202(1)$ & $37(1)$ \\
\hline$C(385)$ & $10096(2)$ & $-1969(2)$ & $6151(1)$ & $36(1)$ \\
\hline C(386) & $9323(2)$ & $-2048(2)$ & $6078(1)$ & $29(1)$ \\
\hline$C(1 S)$ & $1442(3)$ & 7963(3) & $5152(2)$ & $101(2)$ \\
\hline $\mathrm{Cl}(11)$ & 1821(1) & $7473(1)$ & $4688(1)$ & $105(1)$ \\
\hline $\mathrm{Cl}(12)$ & $2150(1)$ & $8246(1)$ & $5684(1)$ & $116(1)$ \\
\hline$C(2 S)$ & $870(3)$ & $4449(2)$ & $3398(2)$ & $94(2)$ \\
\hline $\mathrm{Cl}(21)$ & 994(1) & $5098(1)$ & $3905(1)$ & $136(1)$ \\
\hline $\mathrm{Cl}(22)$ & $109(1)$ & $3842(1)$ & $3331(1)$ & $119(1)$ \\
\hline $\mathrm{C}(3 \mathrm{~S})$ & $8839(3)$ & $6976(3)$ & $8847(3)$ & $215(5)$ \\
\hline $\mathrm{Cl}(31)$ & $9705(1)$ & $6737(1)$ & $9058(1)$ & $194(1)$ \\
\hline $\mathrm{Cl}(32)$ & $8145(1)$ & $6338(1)$ & $8768(1)$ & $204(1)$ \\
\hline$C(6 S)$ & $2448(2)$ & $3505(2)$ & $2744(2)$ & $79(1)$ \\
\hline $\mathrm{Cl}(61)$ & $1625(3)$ & $3884(3)$ & 2461(2) & $211(2)$ \\
\hline $\mathrm{Cl}(62)$ & 2733(2) & $2815(2)$ & $2348(1)$ & $85(1)$ \\
\hline $\mathrm{Cl}(63)$ & $2018(1)$ & $4264(1)$ & $2534(1)$ & $89(1)$ \\
\hline $\mathrm{Cl}(64)$ & $1745(2)$ & 2831(2) & $2432(2)$ & $122(1)$ \\
\hline $\mathrm{Cl}(65)$ & $2306(2)$ & $2713(2)$ & 2401(1) & $57(1)$ \\
\hline $\mathrm{C}(11 \mathrm{~S})$ & $3189(1)$ & $6868(1)$ & $2975(1)$ & $122(2)$ \\
\hline $\mathrm{C}(12 \mathrm{~S})$ & $2835(2)$ & $7462(2)$ & $3156(1)$ & 241(3) \\
\hline$C(13 S)$ & $2278(2)$ & $7361(2)$ & $3410(1)$ & $158(3)$ \\
\hline
\end{tabular}




\begin{tabular}{|c|c|c|c|c|}
\hline $\mathrm{C}(14 \mathrm{~S})$ & $2075(2)$ & $6666(3)$ & $3483(1)$ & 131(2) \\
\hline$C(15 S)$ & $2430(2)$ & $6071(2)$ & $3302(1)$ & 152(3) \\
\hline$C(16 S)$ & 2986(2) & $6172(2)$ & $3048(1)$ & $149(3)$ \\
\hline $\mathrm{C}(4 \mathrm{~S})$ & $8956(4)$ & $4150(4)$ & $9449(2)$ & $135(3)$ \\
\hline $\mathrm{Cl}(41)$ & $9032(2)$ & $4197(1)$ & $10045(1)$ & $135(1)$ \\
\hline $\mathrm{Cl}(42)$ & $8459(2)$ & $4630(2)$ & $9102(1)$ & $163(1)$ \\
\hline $\mathrm{C}(21 \mathrm{~S})$ & $6165(4)$ & $-58(4)$ & $9773(2)$ & $87(2)$ \\
\hline $\mathrm{C}(22 \mathrm{~S})$ & $6439(5)$ & $332(5)$ & $9373(2)$ & 161(4) \\
\hline $\mathrm{C}(23 \mathrm{~S})$ & $6288(4)$ & $-44(5)$ & $8828(2)$ & 153(4) \\
\hline $\mathrm{C}(24 \mathrm{~S})$ & $5639(4)$ & $286(3)$ & $8528(2)$ & $170(5)$ \\
\hline$C(25 S)$ & $6068(5)$ & 938(4) & $8421(3)$ & $182(5)$ \\
\hline$C(26 S)$ & $5553(5)$ & $1273(4)$ & 7997(3) & $166(5)$ \\
\hline$C(5 S)$ & $6097(4)$ & $-1227(4)$ & $9872(3)$ & $48(3)$ \\
\hline $\mathrm{Cl}(51)$ & $5430(2)$ & $-828(1)$ & $10122(1)$ & $79(1)$ \\
\hline $\mathrm{Cl}(52)$ & $5570(1)$ & $-1730(1)$ & $9306(1)$ & $72(1)$ \\
\hline $\mathrm{C}(31 \mathrm{~S})$ & $7746(3)$ & $3563(5)$ & $9503(4)$ & $125(5)$ \\
\hline $\mathrm{C}(32 \mathrm{~S})$ & 6943(3) & $3377(3)$ & $9597(2)$ & $48(2)$ \\
\hline $\mathrm{C}(33 \mathrm{~S})$ & $6708(5)$ & 2591(4) & $9283(2)$ & $278(12)$ \\
\hline $\mathrm{C}(34 \mathrm{~S})$ & $6130(5)$ & $2679(5)$ & $8808(2)$ & 205(9) \\
\hline $\mathrm{C}(35 \mathrm{~S})$ & $6339(5)$ & $2104(5)$ & $8381(2)$ & $127(5)$ \\
\hline $\mathrm{C}(36 \mathrm{~S})$ & $5592(2)$ & $1879(2)$ & $8006(1)$ & $115(5)$ \\
\hline $\mathrm{O}(1 \mathrm{~W})$ & $5348(2)$ & $-1442(2)$ & $9623(1)$ & $98(2)$ \\
\hline $\mathrm{O}(2 \mathrm{~W})$ & 6891(2) & $3022(2)$ & $9328(1)$ & $114(2)$ \\
\hline $\mathrm{O}(3 \mathrm{~W})$ & $6217(2)$ & $1903(2)$ & $9527(1)$ & $132(3)$ \\
\hline
\end{tabular}


Table S2. Bond lengths $[\AA ̊]$ and angles $\left[{ }^{\circ}\right]$ for 2,4,6-[4-(Cl(dppe $\left.\left.)_{2} \mathrm{RuC} \equiv \mathrm{C}\right) \mathrm{C}_{6} \mathrm{H}_{4}\right]_{3}-1,3,5-\mathrm{C}_{3} \mathrm{~N}_{3}(\mathbf{5})$.

\begin{tabular}{|c|c|c|c|}
\hline $\mathrm{Ru}(1)-\mathrm{C}(19)$ & $2.000(3)$ & $\mathrm{Ru}(1)-\mathrm{P}(13)$ & $2.3479(9)$ \\
\hline $\mathrm{Ru}(1)-\mathrm{P}(12)$ & $2.3723(8)$ & $\mathrm{Ru}(1)-\mathrm{P}(11)$ & $2.3930(9)$ \\
\hline $\mathrm{Ru}(1)-\mathrm{Cl}(1)$ & $2.4708(8)$ & $\mathrm{Ru}(2)-\mathrm{C}(29)$ & $1.979(2)$ \\
\hline $\mathrm{Ru}(2)-\mathrm{P}(22)$ & $2.3568(9)$ & $\mathrm{Ru}(2)-\mathrm{P}(23)$ & $2.3606(9)$ \\
\hline $\mathrm{Ru}(2)-\mathrm{P}(24)$ & $2.3737(9)$ & $\mathrm{Ru}(2)-\mathrm{P}(21)$ & $2.3742(10)$ \\
\hline $\mathrm{Ru}(2)-\mathrm{Cl}(2)$ & $2.4956(8)$ & $\mathrm{Ru}(3)-\mathrm{C}(39)$ & $1.987(2)$ \\
\hline $\mathrm{Ru}(3)-\mathrm{P}(32)$ & $2.3548(7)$ & $\mathrm{Ru}(3)-\mathrm{P}(33)$ & $2.3550(9)$ \\
\hline $\mathrm{Ru}(3)-\mathrm{P}(34)$ & $2.3655(8)$ & $\mathrm{Ru}(3)-\mathrm{P}(31)$ & $2.3727(9)$ \\
\hline $\mathrm{Ru}(3)-\mathrm{Cl}(3)$ & $2.5111(8)$ & $\mathrm{P}(11)-\mathrm{C}(101)$ & $1.829(3)$ \\
\hline $\mathrm{P}(11)-\mathrm{C}(111)$ & $1.831(3)$ & $\mathrm{P}(11)-\mathrm{C}(121)$ & $1.850(3)$ \\
\hline $\mathrm{P}(12)-\mathrm{C}(102)$ & $1.830(3)$ & $\mathrm{P}(12)-\mathrm{C}(131)$ & $1.831(3)$ \\
\hline $\mathrm{P}(12)-\mathrm{C}(141)$ & $1.837(3)$ & $\mathrm{P}(13)-\mathrm{C}(161)$ & $1.822(3)$ \\
\hline $\mathrm{P}(13)-\mathrm{C}(151)$ & $1.839(3)$ & $\mathrm{P}(13)-\mathrm{C}(103)$ & $1.844(3)$ \\
\hline $\mathrm{P}(14)-\mathrm{C}(181)$ & $1.811(3)$ & $\mathrm{P}(14)-\mathrm{C}(171)$ & $1.824(3)$ \\
\hline $\mathrm{P}(14)-\mathrm{C}(104)$ & $1.845(3)$ & $\mathrm{P}(21)-\mathrm{C}(211)$ & $1.818(3)$ \\
\hline $\mathrm{P}(21)-\mathrm{C}(201)$ & $1.836(3)$ & $\mathrm{P}(21)-\mathrm{C}(221)$ & $1.839(3)$ \\
\hline $\mathrm{P}(22)-\mathrm{C}(231)$ & $1.829(3)$ & $\mathrm{P}(22)-\mathrm{C}(241)$ & $1.830(3)$ \\
\hline $\mathrm{P}(22)-\mathrm{C}(202)$ & $1.844(3)$ & $\mathrm{P}(23)-\mathrm{C}(203)$ & $1.825(3)$ \\
\hline $\mathrm{P}(23)-\mathrm{C}(251)$ & $1.830(3)$ & $\mathrm{P}(23)-\mathrm{C}(261)$ & $1.842(3)$ \\
\hline $\mathrm{P}(24)-\mathrm{C}(281)$ & $1.824(3)$ & $\mathrm{P}(24)-\mathrm{C}(271)$ & $1.835(3)$ \\
\hline P(24)-C(204) & $1.864(3)$ & $\mathrm{P}(31)-\mathrm{C}(321)$ & $1.829(3)$ \\
\hline $\mathrm{P}(31)-\mathrm{C}(311)$ & $1.850(3)$ & $\mathrm{P}(31)-\mathrm{C}(301)$ & $1.864(2)$ \\
\hline $\mathrm{P}(32)-\mathrm{C}(341)$ & $1.832(3)$ & $\mathrm{P}(32)-\mathrm{C}(302)$ & $1.853(3)$ \\
\hline $\mathrm{P}(32)-\mathrm{C}(331)$ & $1.855(3)$ & $\mathrm{P}(33)-\mathrm{C}(361)$ & $1.819(3)$ \\
\hline $\mathrm{P}(33)-\mathrm{C}(351)$ & $1.849(3)$ & $\mathrm{P}(33)-\mathrm{C}(303)$ & $1.869(2)$ \\
\hline $\mathrm{P}(34)-\mathrm{C}(381)$ & $1.829(3)$ & $\mathrm{P}(34)-\mathrm{C}(304)$ & $1.837(3)$ \\
\hline $\mathrm{P}(34)-\mathrm{C}(371)$ & $1.845(3)$ & $\mathrm{N}(1)-\mathrm{C}(11)$ & $1.333(3)$ \\
\hline $\mathrm{N}(1)-\mathrm{C}(31)$ & $1.351(3)$ & $\mathrm{N}(2)-\mathrm{C}(21)$ & $1.318(3)$ \\
\hline $\mathrm{N}(2)-\mathrm{C}(11)$ & $1.343(3)$ & $\mathrm{N}(3)-\mathrm{C}(31)$ & $1.339(3)$ \\
\hline $\mathrm{N}(3)-\mathrm{C}(21)$ & $1.349(3)$ & $\mathrm{C}(11)-\mathrm{C}(12)$ & $1.493(4)$ \\
\hline$C(12)-C(13)$ & $1.381(3)$ & $\mathrm{C}(12)-\mathrm{C}(17)$ & $1.405(4)$ \\
\hline$C(13)-C(14)$ & $1.372(4)$ & $\mathrm{C}(14)-\mathrm{C}(15)$ & $1.410(4)$ \\
\hline$C(15)-C(16)$ & $1.377(3)$ & $\mathrm{C}(15)-\mathrm{C}(18)$ & $1.453(4)$ \\
\hline$C(16)-C(17)$ & $1.379(4)$ & $\mathrm{C}(18)-\mathrm{C}(19)$ & $1.187(4)$ \\
\hline$C(21)-C(22)$ & $1.476(3)$ & $\mathrm{C}(22)-\mathrm{C}(23)$ & $1.380(4)$ \\
\hline$C(22)-C(27)$ & $1.388(4)$ & $\mathrm{C}(23)-\mathrm{C}(24)$ & $1.384(4)$ \\
\hline
\end{tabular}




\begin{tabular}{|c|c|c|c|}
\hline $\mathrm{C}(24)-\mathrm{C}(25)$ & $1.396(4)$ & $C(25)-C(26)$ & $1.392(4)$ \\
\hline$C(25)-C(28)$ & $1.450(4)$ & $C(26)-C(27)$ & $1.370(3)$ \\
\hline $\mathrm{C}(28)-\mathrm{C}(29)$ & $1.181(4)$ & $C(31)-C(32)$ & $1.457(4)$ \\
\hline$C(32)-C(37)$ & $1.387(3)$ & $C(32)-C(33)$ & $1.404(4)$ \\
\hline$C(33)-C(34)$ & $1.369(4)$ & $C(34)-C(35)$ & $1.400(4)$ \\
\hline$C(35)-C(36)$ & $1.416(4)$ & $\mathrm{C}(35)-\mathrm{C}(38)$ & $1.448(4)$ \\
\hline$C(36)-C(37)$ & $1.381(4)$ & $\mathrm{C}(38)-\mathrm{C}(39)$ & $1.205(4)$ \\
\hline$C(101)-C(102)$ & $1.528(4)$ & $C(103)-C(104)$ & $1.511(4)$ \\
\hline$C(111)-C(116)$ & $1.375(4)$ & $\mathrm{C}(111)-\mathrm{C}(112)$ & $1.398(4)$ \\
\hline$C(112)-C(113)$ & $1.406(4)$ & $C(113)-C(114)$ & $1.366(5)$ \\
\hline$C(114)-C(115)$ & $1.382(5)$ & $\mathrm{C}(115)-\mathrm{C}(116)$ & $1.400(4)$ \\
\hline$C(121)-C(122)$ & $1.375(4)$ & $\mathrm{C}(121)-\mathrm{C}(126)$ & $1.398(4)$ \\
\hline$C(122)-C(123)$ & $1.398(4)$ & $\mathrm{C}(123)-\mathrm{C}(124)$ & $1.396(5)$ \\
\hline$C(124)-C(125)$ & $1.368(5)$ & $C(125)-C(126)$ & $1.385(4)$ \\
\hline$C(131)-C(132)$ & $1.354(4)$ & $\mathrm{C}(131)-\mathrm{C}(136)$ & $1.381(4)$ \\
\hline$C(132)-C(133)$ & $1.378(5)$ & $\mathrm{C}(133)-\mathrm{C}(134)$ & $1.399(5)$ \\
\hline$C(134)-C(135)$ & $1.363(5)$ & $C(135)-C(136)$ & $1.377(5)$ \\
\hline$C(141)-C(142)$ & $1.395(4)$ & $C(141)-C(146)$ & $1.407(4)$ \\
\hline$C(142)-C(143)$ & $1.378(4)$ & $\mathrm{C}(143)-\mathrm{C}(144)$ & $1.354(5)$ \\
\hline$C(144)-C(145)$ & $1.385(5)$ & $\mathrm{C}(145)-\mathrm{C}(146)$ & $1.380(4)$ \\
\hline$C(151)-C(152)$ & $1.385(4)$ & $\mathrm{C}(151)-\mathrm{C}(156)$ & $1.424(4)$ \\
\hline$C(152)-C(153)$ & $1.411(4)$ & $\mathrm{C}(153)-\mathrm{C}(154)$ & $1.376(5)$ \\
\hline$C(154)-C(155)$ & $1.395(4)$ & $\mathrm{C}(155)-\mathrm{C}(156)$ & $1.368(4)$ \\
\hline$C(161)-C(162)$ & $1.376(4)$ & $C(161)-C(166)$ & $1.391(4)$ \\
\hline$C(162)-C(163)$ & $1.364(4)$ & $C(163)-C(164)$ & $1.398(5)$ \\
\hline$C(164)-C(165)$ & $1.363(5)$ & $\mathrm{C}(165)-\mathrm{C}(166)$ & $1.374(4)$ \\
\hline $\mathrm{C}(171)-\mathrm{C}(176)$ & $1.388(4)$ & $\mathrm{C}(171)-\mathrm{C}(172)$ & $1.402(4)$ \\
\hline$C(172)-C(173)$ & $1.380(5)$ & $\mathrm{C}(173)-\mathrm{C}(174)$ & $1.398(5)$ \\
\hline$C(174)-C(175)$ & $1.343(5)$ & $\mathrm{C}(175)-\mathrm{C}(176)$ & $1.373(4)$ \\
\hline$C(181)-C(186)$ & $1.360(5)$ & $\mathrm{C}(181)-\mathrm{C}(182)$ & $1.412(4)$ \\
\hline$C(182)-C(183)$ & $1.375(5)$ & $\mathrm{C}(183)-\mathrm{C}(184)$ & $1.361(6)$ \\
\hline$C(184)-C(185)$ & $1.338(5)$ & $\mathrm{C}(185)-\mathrm{C}(186)$ & $1.468(5)$ \\
\hline $\mathrm{C}(201)-\mathrm{C}(202)$ & $1.529(4)$ & $C(203)-C(204)$ & $1.507(4)$ \\
\hline$C(211)-C(216)$ & $1.413(4)$ & $\mathrm{C}(211)-\mathrm{C}(212)$ & $1.417(4)$ \\
\hline$C(212)-C(213)$ & $1.357(5)$ & $\mathrm{C}(213)-\mathrm{C}(214)$ & $1.392(4)$ \\
\hline$C(214)-C(215)$ & $1.374(5)$ & $C(215)-C(216)$ & $1.369(4)$ \\
\hline$C(221)-C(226)$ & $1.364(5)$ & $\mathrm{C}(221)-\mathrm{C}(222)$ & $1.372(4)$ \\
\hline $\mathrm{C}(222)-\mathrm{C}(223)$ & $1.380(4)$ & $\mathrm{C}(223)-\mathrm{C}(224)$ & $1.403(5)$ \\
\hline
\end{tabular}




\begin{tabular}{|c|c|c|c|}
\hline $\mathrm{C}(224)-\mathrm{C}(225)$ & $1.332(6)$ & $\mathrm{C}(225)-\mathrm{C}(226)$ & $1.386(5)$ \\
\hline$C(231)-C(236)$ & $1.404(4)$ & $\mathrm{C}(231)-\mathrm{C}(232)$ & $1.412(4)$ \\
\hline $\mathrm{C}(232)-\mathrm{C}(233)$ & $1.380(4)$ & $\mathrm{C}(233)-\mathrm{C}(234)$ & $1.370(5)$ \\
\hline $\mathrm{C}(234)-\mathrm{C}(235)$ & $1.373(4)$ & $\mathrm{C}(235)-\mathrm{C}(236)$ & $1.392(4)$ \\
\hline$C(241)-C(242)$ & $1.387(4)$ & $C(241)-C(246)$ & $1.407(4)$ \\
\hline $\mathrm{C}(242)-\mathrm{C}(243)$ & $1.376(4)$ & $\mathrm{C}(243)-\mathrm{C}(244)$ & $1.348(5)$ \\
\hline$C(244)-C(245)$ & $1.361(5)$ & $C(245)-C(246)$ & $1.409(4)$ \\
\hline $\mathrm{C}(251)-\mathrm{C}(256)$ & $1.383(4)$ & $\mathrm{C}(251)-\mathrm{C}(252)$ & $1.394(4)$ \\
\hline$C(252)-C(253)$ & $1.408(4)$ & $C(253)-C(254)$ & $1.354(4)$ \\
\hline$C(254)-C(255)$ & $1.377(4)$ & $C(255)-C(256)$ & $1.408(4)$ \\
\hline$C(261)-C(262)$ & $1.397(4)$ & $C(261)-C(266)$ & $1.400(4)$ \\
\hline $\mathrm{C}(262)-\mathrm{C}(263)$ & $1.380(4)$ & $C(263)-C(264)$ & $1.358(4)$ \\
\hline$C(264)-C(265)$ & $1.394(5)$ & $C(265)-C(266)$ & $1.390(4)$ \\
\hline$C(271)-C(276)$ & $1.382(4)$ & $\mathrm{C}(271)-\mathrm{C}(272)$ & $1.397(4)$ \\
\hline $\mathrm{C}(272)-\mathrm{C}(273)$ & $1.383(4)$ & $\mathrm{C}(273)-\mathrm{C}(274)$ & $1.380(5)$ \\
\hline $\mathrm{C}(274)-\mathrm{C}(275)$ & $1.378(4)$ & $\mathrm{C}(275)-\mathrm{C}(276)$ & $1.399(4)$ \\
\hline $\mathrm{C}(281)-\mathrm{C}(286)$ & $1.402(4)$ & $\mathrm{C}(281)-\mathrm{C}(282)$ & $1.417(4)$ \\
\hline $\mathrm{C}(282)-\mathrm{C}(283)$ & $1.377(4)$ & $\mathrm{C}(283)-\mathrm{C}(284)$ & $1.375(4)$ \\
\hline $\mathrm{C}(284)-\mathrm{C}(285)$ & $1.404(5)$ & $\mathrm{C}(285)-\mathrm{C}(286)$ & $1.350(4)$ \\
\hline $\mathrm{C}(301)-\mathrm{C}(302)$ & $1.536(4)$ & $\mathrm{C}(303)-\mathrm{C}(304)$ & $1.523(4)$ \\
\hline $\mathrm{C}(311)-\mathrm{C}(312)$ & $1.385(4)$ & $\mathrm{C}(311)-\mathrm{C}(316)$ & $1.389(5)$ \\
\hline $\mathrm{C}(312)-\mathrm{C}(313)$ & $1.395(4)$ & $\mathrm{C}(313)-\mathrm{C}(314)$ & $1.366(5)$ \\
\hline $\mathrm{C}(314)-\mathrm{C}(315)$ & $1.369(5)$ & $C(315)-C(316)$ & $1.376(5)$ \\
\hline $\mathrm{C}(321)-\mathrm{C}(322)$ & $1.405(4)$ & $\mathrm{C}(321)-\mathrm{C}(326)$ & $1.415(4)$ \\
\hline $\mathrm{C}(322)-\mathrm{C}(323)$ & $1.412(4)$ & $\mathrm{C}(323)-\mathrm{C}(324)$ & $1.379(4)$ \\
\hline $\mathrm{C}(324)-\mathrm{C}(325)$ & $1.367(4)$ & $C(325)-C(326)$ & $1.382(4)$ \\
\hline $\mathrm{C}(331)-\mathrm{C}(336)$ & $1.380(4)$ & $\mathrm{C}(331)-\mathrm{C}(332)$ & $1.407(3)$ \\
\hline $\mathrm{C}(332)-\mathrm{C}(333)$ & $1.378(4)$ & $\mathrm{C}(333)-\mathrm{C}(334)$ & $1.385(4)$ \\
\hline $\mathrm{C}(334)-\mathrm{C}(335)$ & $1.385(4)$ & $\mathrm{C}(335)-\mathrm{C}(336)$ & $1.390(4)$ \\
\hline $\mathrm{C}(341)-\mathrm{C}(346)$ & $1.377(4)$ & $\mathrm{C}(341)-\mathrm{C}(342)$ & $1.390(4)$ \\
\hline $\mathrm{C}(342)-\mathrm{C}(343)$ & $1.372(4)$ & $\mathrm{C}(343)-\mathrm{C}(344)$ & $1.353(4)$ \\
\hline $\mathrm{C}(344)-\mathrm{C}(345)$ & $1.392(4)$ & $C(345)-C(346)$ & $1.374(4)$ \\
\hline $\mathrm{C}(351)-\mathrm{C}(352)$ & $1.368(3)$ & $C(351)-C(356)$ & $1.399(4)$ \\
\hline $\mathrm{C}(352)-\mathrm{C}(353)$ & $1.389(4)$ & $C(353)-C(354)$ & $1.376(4)$ \\
\hline $\mathrm{C}(354)-\mathrm{C}(355)$ & $1.369(4)$ & $C(355)-C(356)$ & $1.402(4)$ \\
\hline $\mathrm{C}(361)-\mathrm{C}(366)$ & $1.399(4)$ & $C(361)-C(362)$ & $1.400(4)$ \\
\hline $\mathrm{C}(362)-\mathrm{C}(363)$ & $1.360(4)$ & $\mathrm{C}(363)-\mathrm{C}(364)$ & $1.360(4)$ \\
\hline $\mathrm{C}(364)-\mathrm{C}(365)$ & $1.390(4)$ & $\mathrm{C}(365)-\mathrm{C}(366)$ & $1.363(4)$ \\
\hline
\end{tabular}




\begin{tabular}{|c|c|c|c|}
\hline C(371)-C(372) & $1.370(4)$ & $\mathrm{C}(371)-\mathrm{C}(376)$ & $1.376(4)$ \\
\hline $\mathrm{C}(372)-\mathrm{C}(373)$ & $1.373(4)$ & $\mathrm{C}(373)-\mathrm{C}(374)$ & $1.399(4)$ \\
\hline C(374)-C(375) & $1.372(4)$ & $C(375)-C(376)$ & $1.404(4)$ \\
\hline C(381)-C(386) & $1.395(4)$ & $\mathrm{C}(381)-\mathrm{C}(382)$ & $1.405(4)$ \\
\hline $\mathrm{C}(382)-\mathrm{C}(383)$ & $1.386(4)$ & $\mathrm{C}(383)-\mathrm{C}(384)$ & $1.372(4)$ \\
\hline C(384)-C(385) & $1.370(4)$ & C(385)-C(386) & $1.382(4)$ \\
\hline $\mathrm{C}(1 \mathrm{~S})-\mathrm{Cl}(12)$ & $1.750(4)$ & $\mathrm{C}(1 \mathrm{~S})-\mathrm{Cl}(11)$ & $1.770(5)$ \\
\hline $\mathrm{C}(2 \mathrm{~S})-\mathrm{Cl}(21)$ & $1.714(5)$ & $\mathrm{C}(2 \mathrm{~S})-\mathrm{Cl}(22)$ & $1.739(5)$ \\
\hline $\mathrm{C}(3 \mathrm{~S})-\mathrm{Cl}(31)$ & $1.645(5)$ & $\mathrm{C}(3 \mathrm{~S})-\mathrm{Cl}(32)$ & $1.680(6)$ \\
\hline $\mathrm{C}(6 \mathrm{~S})-\mathrm{Cl}(65)$ & $1.639(5)$ & $\mathrm{C}(6 \mathrm{~S})-\mathrm{Cl}(63)$ & $1.724(4)$ \\
\hline $\mathrm{C}(6 \mathrm{~S})-\mathrm{Cl}(61)$ & $1.732(6)$ & $\mathrm{C}(6 \mathrm{~S})-\mathrm{Cl}(62)$ & $1.756(5)$ \\
\hline $\mathrm{C}(6 \mathrm{~S})-\mathrm{Cl}(64)$ & $1.776(5)$ & $\mathrm{C}(11 \mathrm{~S})-\mathrm{C}(12 \mathrm{~S})$ & 1.3900 \\
\hline$C(11 S)-C(16 S)$ & 1.3900 & $\mathrm{C}(12 \mathrm{~S})-\mathrm{C}(13 \mathrm{~S})$ & 1.3900 \\
\hline $\mathrm{C}(13 \mathrm{~S})-\mathrm{C}(14 \mathrm{~S})$ & 1.3900 & $\mathrm{C}(14 \mathrm{~S})-\mathrm{C}(15 \mathrm{~S})$ & 1.3900 \\
\hline$C(15 S)-C(16 S)$ & 1.3900 & $\mathrm{C}(4 \mathrm{~S})-\mathrm{Cl}(42)$ & $1.546(7)$ \\
\hline $\mathrm{C}(4 \mathrm{~S})-\mathrm{Cl}(41)$ & $1.630(7)$ & $\mathrm{C}(21 \mathrm{~S})-\mathrm{C}(22 \mathrm{~S})$ & $1.570(9)$ \\
\hline $\mathrm{C}(22 \mathrm{~S})-\mathrm{C}(23 \mathrm{~S})$ & $1.556(8)$ & $\mathrm{C}(23 \mathrm{~S})-\mathrm{C}(24 \mathrm{~S})$ & $1.473(9)$ \\
\hline $\mathrm{C}(24 \mathrm{~S})-\mathrm{C}(25 \mathrm{~S})$ & $1.529(10)$ & $\mathrm{C}(25 \mathrm{~S})-\mathrm{C}(26 \mathrm{~S})$ & $1.535(10)$ \\
\hline $\mathrm{C}(5 \mathrm{~S})-\mathrm{Cl}(51)$ & $1.679(8)$ & $\mathrm{C}(5 \mathrm{~S})-\mathrm{Cl}(52)$ & $1.793(7)$ \\
\hline $\mathrm{C}(31 \mathrm{~S})-\mathrm{C}(32 \mathrm{~S})$ & $1.590(9)$ & $\mathrm{C}(32 \mathrm{~S})-\mathrm{C}(33 \mathrm{~S})$ & $1.608(8)$ \\
\hline C(33S)-C(34S) & $1.523(9)$ & $\mathrm{C}(34 \mathrm{~S})-\mathrm{C}(35 \mathrm{~S})$ & $1.618(10)$ \\
\hline$C(35 S)-C(36 S)$ & $1.534(7)$ & & \\
\hline C(19)-Ru(1)-P(13) & $81.77(8)$ & $\mathrm{C}(19)-\mathrm{Ru}(1)-\mathrm{P}(14)$ & $84.39(7)$ \\
\hline $\mathrm{P}(13)-\mathrm{Ru}(1)-\mathrm{P}(14)$ & $82.77(3)$ & $\mathrm{C}(19)-\mathrm{Ru}(1)-\mathrm{P}(12)$ & $95.63(7)$ \\
\hline $\mathrm{P}(13)-\mathrm{Ru}(1)-\mathrm{P}(12)$ & $95.29(3)$ & $\mathrm{P}(14)-\mathrm{Ru}(1)-\mathrm{P}(12)$ & $178.04(3)$ \\
\hline $\mathrm{C}(19)-\mathrm{Ru}(1)-\mathrm{P}(11)$ & $97.03(8)$ & $\mathrm{P}(13)-\mathrm{Ru}(1)-\mathrm{P}(11)$ & $177.56(3)$ \\
\hline $\mathrm{P}(14)-\mathrm{Ru}(1)-\mathrm{P}(11)$ & $99.25(3)$ & $\mathrm{P}(12)-\mathrm{Ru}(1)-\mathrm{P}(11)$ & $82.69(3)$ \\
\hline $\mathrm{C}(19)-\mathrm{Ru}(1)-\mathrm{Cl}(1)$ & $174.07(8)$ & $\mathrm{P}(13)-\mathrm{Ru}(1)-\mathrm{Cl}(1)$ & $102.06(3)$ \\
\hline $\mathrm{P}(14)-\mathrm{Ru}(1)-\mathrm{Cl}(1)$ & $91.57(3)$ & $\mathrm{P}(12)-\mathrm{Ru}(1)-\mathrm{Cl}(1)$ & $88.56(3)$ \\
\hline $\mathrm{P}(11)-\mathrm{Ru}(1)-\mathrm{Cl}(1)$ & $79.31(3)$ & $\mathrm{C}(29)-\mathrm{Ru}(2)-\mathrm{P}(22)$ & $88.41(8)$ \\
\hline $\mathrm{C}(29)-\mathrm{Ru}(2)-\mathrm{P}(23)$ & $97.44(8)$ & $\mathrm{P}(22)-\mathrm{Ru}(2)-\mathrm{P}(23)$ & $95.79(3)$ \\
\hline $\mathrm{C}(29)-\mathrm{Ru}(2)-\mathrm{P}(24)$ & $92.81(8)$ & $\mathrm{P}(22)-\mathrm{Ru}(2)-\mathrm{P}(24)$ & $176.18(3)$ \\
\hline $\mathrm{P}(23)-\mathrm{Ru}(2)-\mathrm{P}(24)$ & $80.46(3)$ & $\mathrm{C}(29)-\mathrm{Ru}(2)-\mathrm{P}(21)$ & $82.99(8)$ \\
\hline $\mathrm{P}(22)-\mathrm{Ru}(2)-\mathrm{P}(21)$ & $83.96(3)$ & $\mathrm{P}(23)-\mathrm{Ru}(2)-\mathrm{P}(21)$ & $179.50(3)$ \\
\hline $\mathrm{P}(24)-\mathrm{Ru}(2)-\mathrm{P}(21)$ & $99.78(3)$ & $\mathrm{C}(29)-\mathrm{Ru}(2)-\mathrm{Cl}(2)$ & $175.93(8)$ \\
\hline $\mathrm{P}(22)-\mathrm{Ru}(2)-\mathrm{Cl}(2)$ & $88.06(3)$ & $\mathrm{P}(23)-\mathrm{Ru}(2)-\mathrm{Cl}(2)$ & $84.92(3)$ \\
\hline $\mathrm{P}(24)-\mathrm{Ru}(2)-\mathrm{Cl}(2)$ & $90.85(3)$ & $\mathrm{P}(21)-\mathrm{Ru}(2)-\mathrm{Cl}(2)$ & $94.64(3)$ \\
\hline
\end{tabular}




\begin{tabular}{|c|c|c|c|}
\hline C(39)-Ru(3)-P(32) & $82.60(7)$ & $\mathrm{C}(39)-\mathrm{Ru}(3)-\mathrm{P}(33)$ & $91.43(8)$ \\
\hline $\mathrm{P}(32)-\mathrm{Ru}(3)-\mathrm{P}(33)$ & $96.34(3)$ & $\mathrm{C}(39)-\mathrm{Ru}(3)-\mathrm{P}(34)$ & $90.01(7)$ \\
\hline $\mathrm{P}(32)-\mathrm{Ru}(3)-\mathrm{P}(34)$ & $172.49(3)$ & $\mathrm{P}(33)-\mathrm{Ru}(3)-\mathrm{P}(34)$ & $82.43(3)$ \\
\hline $\mathrm{C}(39)-\mathrm{Ru}(3)-\mathrm{P}(31)$ & $86.15(8)$ & $\mathrm{P}(32)-\mathrm{Ru}(3)-\mathrm{P}(31)$ & $82.43(3)$ \\
\hline $\mathrm{P}(33)-\mathrm{Ru}(3)-\mathrm{P}(31)$ & $177.41(3)$ & $\mathrm{P}(34)-\mathrm{Ru}(3)-\mathrm{P}(31)$ & $98.49(3)$ \\
\hline $\mathrm{C}(39)-\mathrm{Ru}(3)-\mathrm{Cl}(3)$ & $173.51(8)$ & $\mathrm{P}(32)-\mathrm{Ru}(3)-\mathrm{Cl}(3)$ & $101.49(3)$ \\
\hline $\mathrm{P}(33)-\mathrm{Ru}(3)-\mathrm{Cl}(3)$ & $83.15(3)$ & $\mathrm{P}(34)-\mathrm{Ru}(3)-\mathrm{Cl}(3)$ & $85.75(3)$ \\
\hline $\mathrm{P}(31)-\mathrm{Ru}(3)-\mathrm{Cl}(3)$ & $99.32(3)$ & $\mathrm{C}(101)-\mathrm{P}(11)-\mathrm{C}(111)$ & $103.73(13)$ \\
\hline $\mathrm{C}(101)-\mathrm{P}(11)-\mathrm{C}(121)$ & $97.42(12)$ & $\mathrm{C}(111)-\mathrm{P}(11)-\mathrm{C}(121)$ & $100.69(13)$ \\
\hline $\mathrm{C}(101)-\mathrm{P}(11)-\mathrm{Ru}(1)$ & $104.23(10)$ & $\mathrm{C}(111)-\mathrm{P}(11)-\mathrm{Ru}(1)$ & $122.16(9)$ \\
\hline $\mathrm{C}(121)-\mathrm{P}(11)-\mathrm{Ru}(1)$ & $124.23(10)$ & $\mathrm{C}(102)-\mathrm{P}(12)-\mathrm{C}(131)$ & $102.82(14)$ \\
\hline $\mathrm{C}(102)-\mathrm{P}(12)-\mathrm{C}(141)$ & $101.18(13)$ & $\mathrm{C}(131)-\mathrm{P}(12)-\mathrm{C}(141)$ & $103.21(14)$ \\
\hline $\mathrm{C}(102)-\mathrm{P}(12)-\mathrm{Ru}(1)$ & $108.54(9)$ & $\mathrm{C}(131)-\mathrm{P}(12)-\mathrm{Ru}(1)$ & $117.78(9)$ \\
\hline $\mathrm{C}(141)-\mathrm{P}(12)-\mathrm{Ru}(1)$ & $120.75(10)$ & $\mathrm{C}(161)-\mathrm{P}(13)-\mathrm{C}(151)$ & $100.26(12)$ \\
\hline $\mathrm{C}(161)-\mathrm{P}(13)-\mathrm{C}(103)$ & $104.11(13)$ & $\mathrm{C}(151)-\mathrm{P}(13)-\mathrm{C}(103)$ & $98.26(12)$ \\
\hline $\mathrm{C}(161)-\mathrm{P}(13)-\mathrm{Ru}(1)$ & $120.66(9)$ & $\mathrm{C}(151)-\mathrm{P}(13)-\mathrm{Ru}(1)$ & $124.98(10)$ \\
\hline $\mathrm{C}(103)-\mathrm{P}(13)-\mathrm{Ru}(1)$ & $104.76(9)$ & $\mathrm{C}(181)-\mathrm{P}(14)-\mathrm{C}(171)$ & $103.54(13)$ \\
\hline C(181)-P(14)-C(104) & $101.93(13)$ & $\mathrm{C}(171)-\mathrm{P}(14)-\mathrm{C}(104)$ & $102.06(14)$ \\
\hline $\mathrm{C}(181)-\mathrm{P}(14)-\mathrm{Ru}(1)$ & 118.69(10) & $\mathrm{C}(171)-\mathrm{P}(14)-\mathrm{Ru}(1)$ & $119.66(9)$ \\
\hline $\mathrm{C}(104)-\mathrm{P}(14)-\mathrm{Ru}(1)$ & $108.41(9)$ & $\mathrm{C}(211)-\mathrm{P}(21)-\mathrm{C}(201)$ & $101.82(13)$ \\
\hline $\mathrm{C}(211)-\mathrm{P}(21)-\mathrm{C}(221)$ & $100.40(13)$ & $\mathrm{C}(201)-\mathrm{P}(21)-\mathrm{C}(221)$ & $102.91(13)$ \\
\hline $\mathrm{C}(211)-\mathrm{P}(21)-\mathrm{Ru}(2)$ & $121.39(10)$ & $\mathrm{C}(201)-\mathrm{P}(21)-\mathrm{Ru}(2)$ & $105.29(10)$ \\
\hline $\mathrm{C}(221)-\mathrm{P}(21)-\mathrm{Ru}(2)$ & $122.00(11)$ & $\mathrm{C}(231)-\mathrm{P}(22)-\mathrm{C}(241)$ & $101.65(13)$ \\
\hline $\mathrm{C}(231)-\mathrm{P}(22)-\mathrm{C}(202)$ & 101.93(13) & $\mathrm{C}(241)-\mathrm{P}(22)-\mathrm{C}(202)$ & $103.87(13)$ \\
\hline $\mathrm{C}(231)-\mathrm{P}(22)-\mathrm{Ru}(2)$ & $123.20(10)$ & $\mathrm{C}(241)-\mathrm{P}(22)-\mathrm{Ru}(2)$ & $117.09(9)$ \\
\hline $\mathrm{C}(202)-\mathrm{P}(22)-\mathrm{Ru}(2)$ & $106.67(9)$ & $\mathrm{C}(203)-\mathrm{P}(23)-\mathrm{C}(251)$ & $103.75(13)$ \\
\hline $\mathrm{C}(203)-\mathrm{P}(23)-\mathrm{C}(261)$ & $98.43(12)$ & $\mathrm{C}(251)-\mathrm{P}(23)-\mathrm{C}(261)$ & $101.54(12)$ \\
\hline $\mathrm{C}(203)-\mathrm{P}(23)-\mathrm{Ru}(2)$ & $104.16(9)$ & $\mathrm{C}(251)-\mathrm{P}(23)-\mathrm{Ru}(2)$ & $122.67(9)$ \\
\hline $\mathrm{C}(261)-\mathrm{P}(23)-\mathrm{Ru}(2)$ & $122.26(10)$ & $\mathrm{C}(281)-\mathrm{P}(24)-\mathrm{C}(271)$ & $101.19(12)$ \\
\hline $\mathrm{C}(281)-\mathrm{P}(24)-\mathrm{C}(204)$ & $101.54(13)$ & $\mathrm{C}(271)-\mathrm{P}(24)-\mathrm{C}(204)$ & $102.27(12)$ \\
\hline $\mathrm{C}(281)-\mathrm{P}(24)-\mathrm{Ru}(2)$ & $122.66(9)$ & $\mathrm{C}(271)-\mathrm{P}(24)-\mathrm{Ru}(2)$ & $117.42(9)$ \\
\hline $\mathrm{C}(204)-\mathrm{P}(24)-\mathrm{Ru}(2)$ & $108.95(9)$ & $\mathrm{C}(321)-\mathrm{P}(31)-\mathrm{C}(311)$ & $100.01(13)$ \\
\hline $\mathrm{C}(321)-\mathrm{P}(31)-\mathrm{C}(301)$ & $103.57(12)$ & $\mathrm{C}(311)-\mathrm{P}(31)-\mathrm{C}(301)$ & $97.53(12)$ \\
\hline $\mathrm{C}(321)-\mathrm{P}(31)-\mathrm{Ru}(3)$ & $116.26(10)$ & $\mathrm{C}(311)-\mathrm{P}(31)-\mathrm{Ru}(3)$ & $127.71(10)$ \\
\hline $\mathrm{C}(301)-\mathrm{P}(31)-\mathrm{Ru}(3)$ & $108.12(9)$ & $\mathrm{C}(341)-\mathrm{P}(32)-\mathrm{C}(302)$ & $102.46(12)$ \\
\hline $\mathrm{C}(341)-\mathrm{P}(32)-\mathrm{C}(331)$ & $100.93(12)$ & $\mathrm{C}(302)-\mathrm{P}(32)-\mathrm{C}(331)$ & $98.43(12)$ \\
\hline $\mathrm{C}(341)-\mathrm{P}(32)-\mathrm{Ru}(3)$ & $121.37(8)$ & $\mathrm{C}(302)-\mathrm{P}(32)-\mathrm{Ru}(3)$ & $104.07(8)$ \\
\hline $\mathrm{C}(331)-\mathrm{P}(32)-\mathrm{Ru}(3)$ & $125.11(8)$ & $\mathrm{C}(361)-\mathrm{P}(33)-\mathrm{C}(351)$ & $102.04(12)$ \\
\hline
\end{tabular}




\begin{tabular}{|c|c|c|c|}
\hline $\mathrm{C}(361)-\mathrm{P}(33)-\mathrm{C}(303)$ & $101.70(11)$ & $\mathrm{C}(351)-\mathrm{P}(33)-\mathrm{C}(303)$ & $101.97(12)$ \\
\hline $\mathrm{C}(361)-\mathrm{P}(33)-\mathrm{Ru}(3)$ & 119.09(10) & $\mathrm{C}(351)-\mathrm{P}(33)-\mathrm{Ru}(3)$ & $120.46(9)$ \\
\hline $\mathrm{C}(303)-\mathrm{P}(33)-\mathrm{Ru}(3)$ & $108.83(9)$ & $\mathrm{C}(381)-\mathrm{P}(34)-\mathrm{C}(304)$ & $104.01(12)$ \\
\hline $\mathrm{C}(381)-\mathrm{P}(34)-\mathrm{C}(371)$ & $99.87(12)$ & $\mathrm{C}(304)-\mathrm{P}(34)-\mathrm{C}(371)$ & $103.77(13)$ \\
\hline $\mathrm{C}(381)-\mathrm{P}(34)-\mathrm{Ru}(3)$ & $121.47(9)$ & $\mathrm{C}(304)-\mathrm{P}(34)-\mathrm{Ru}(3)$ & $105.20(9)$ \\
\hline $\mathrm{C}(371)-\mathrm{P}(34)-\mathrm{Ru}(3)$ & $120.32(8)$ & $\mathrm{C}(11)-\mathrm{N}(1)-\mathrm{C}(31)$ & $115.0(2)$ \\
\hline $\mathrm{C}(21)-\mathrm{N}(2)-\mathrm{C}(11)$ & $115.6(2)$ & $\mathrm{C}(31)-\mathrm{N}(3)-\mathrm{C}(21)$ & $115.6(2)$ \\
\hline $\mathrm{N}(1)-\mathrm{C}(11)-\mathrm{N}(2)$ & $125.2(2)$ & $\mathrm{N}(1)-\mathrm{C}(11)-\mathrm{C}(12)$ & $118.0(2)$ \\
\hline $\mathrm{N}(2)-\mathrm{C}(11)-\mathrm{C}(12)$ & $116.8(2)$ & $\mathrm{C}(13)-\mathrm{C}(12)-\mathrm{C}(17)$ & $118.7(2)$ \\
\hline$C(13)-C(12)-C(11)$ & $122.1(2)$ & $C(17)-C(12)-C(11)$ & $119.1(2)$ \\
\hline $\mathrm{C}(14)-\mathrm{C}(13)-\mathrm{C}(12)$ & $120.4(2)$ & $\mathrm{C}(13)-\mathrm{C}(14)-\mathrm{C}(15)$ & $121.4(2)$ \\
\hline$C(16)-C(15)-C(14)$ & $117.7(2)$ & $\mathrm{C}(16)-\mathrm{C}(15)-\mathrm{C}(18)$ & $120.7(2)$ \\
\hline $\mathrm{C}(14)-\mathrm{C}(15)-\mathrm{C}(18)$ & $121.6(2)$ & $\mathrm{C}(15)-\mathrm{C}(16)-\mathrm{C}(17)$ & $121.4(2)$ \\
\hline$C(16)-C(17)-C(12)$ & $120.4(2)$ & $\mathrm{C}(19)-\mathrm{C}(18)-\mathrm{C}(15)$ & $177.8(3)$ \\
\hline $\mathrm{C}(18)-\mathrm{C}(19)-\mathrm{Ru}(1)$ & $172.2(2)$ & $\mathrm{N}(2)-\mathrm{C}(21)-\mathrm{N}(3)$ & $124.6(2)$ \\
\hline $\mathrm{N}(2)-\mathrm{C}(21)-\mathrm{C}(22)$ & $117.8(2)$ & $\mathrm{N}(3)-\mathrm{C}(21)-\mathrm{C}(22)$ & $117.6(2)$ \\
\hline $\mathrm{C}(23)-\mathrm{C}(22)-\mathrm{C}(27)$ & $119.2(2)$ & $\mathrm{C}(23)-\mathrm{C}(22)-\mathrm{C}(21)$ & $120.7(2)$ \\
\hline$C(27)-C(22)-C(21)$ & $120.1(2)$ & $\mathrm{C}(22)-\mathrm{C}(23)-\mathrm{C}(24)$ & $120.6(3)$ \\
\hline$C(23)-C(24)-C(25)$ & 120.1(3) & $C(26)-C(25)-C(24)$ & $118.8(2)$ \\
\hline$C(26)-C(25)-C(28)$ & $118.9(2)$ & $\mathrm{C}(24)-\mathrm{C}(25)-\mathrm{C}(28)$ & $122.2(2)$ \\
\hline$C(27)-C(26)-C(25)$ & $120.4(2)$ & $\mathrm{C}(26)-\mathrm{C}(27)-\mathrm{C}(22)$ & $120.8(2)$ \\
\hline $\mathrm{C}(29)-\mathrm{C}(28)-\mathrm{C}(25)$ & $172.4(3)$ & $\mathrm{C}(28)-\mathrm{C}(29)-\mathrm{Ru}(2)$ & $177.7(3)$ \\
\hline $\mathrm{N}(3)-\mathrm{C}(31)-\mathrm{N}(1)$ & $124.0(2)$ & $\mathrm{N}(3)-\mathrm{C}(31)-\mathrm{C}(32)$ & $117.0(2)$ \\
\hline $\mathrm{N}(1)-\mathrm{C}(31)-\mathrm{C}(32)$ & $118.9(2)$ & $\mathrm{C}(37)-\mathrm{C}(32)-\mathrm{C}(33)$ & $117.2(2)$ \\
\hline $\mathrm{C}(37)-\mathrm{C}(32)-\mathrm{C}(31)$ & $121.9(2)$ & $\mathrm{C}(33)-\mathrm{C}(32)-\mathrm{C}(31)$ & $120.8(2)$ \\
\hline$C(34)-C(33)-C(32)$ & $122.7(2)$ & $\mathrm{C}(33)-\mathrm{C}(34)-\mathrm{C}(35)$ & $119.8(3)$ \\
\hline $\mathrm{C}(34)-\mathrm{C}(35)-\mathrm{C}(36)$ & 118.2(2) & $\mathrm{C}(34)-\mathrm{C}(35)-\mathrm{C}(38)$ & $121.6(2)$ \\
\hline $\mathrm{C}(36)-\mathrm{C}(35)-\mathrm{C}(38)$ & $120.2(2)$ & $\mathrm{C}(37)-\mathrm{C}(36)-\mathrm{C}(35)$ & $120.7(2)$ \\
\hline $\mathrm{C}(36)-\mathrm{C}(37)-\mathrm{C}(32)$ & 121.3(3) & $\mathrm{C}(39)-\mathrm{C}(38)-\mathrm{C}(35)$ & 177.1(3) \\
\hline $\mathrm{C}(38)-\mathrm{C}(39)-\mathrm{Ru}(3)$ & $179.7(3)$ & $\mathrm{C}(102)-\mathrm{C}(101)-\mathrm{P}(11)$ & $108.4(2)$ \\
\hline $\mathrm{C}(101)-\mathrm{C}(102)-\mathrm{P}(12)$ & 108.20(18) & $\mathrm{C}(104)-\mathrm{C}(103)-\mathrm{P}(13)$ & $107.3(2)$ \\
\hline $\mathrm{C}(103)-\mathrm{C}(104)-\mathrm{P}(14)$ & $111.59(18)$ & $\mathrm{C}(116)-\mathrm{C}(111)-\mathrm{C}(112)$ & $118.7(3)$ \\
\hline $\mathrm{C}(116)-\mathrm{C}(111)-\mathrm{P}(11)$ & $120.4(2)$ & $\mathrm{C}(112)-\mathrm{C}(111)-\mathrm{P}(11)$ & $120.9(2)$ \\
\hline$C(111)-C(112)-C(113)$ & $118.6(3)$ & $C(114)-C(113)-C(112)$ & $121.7(3)$ \\
\hline $\mathrm{C}(113)-\mathrm{C}(114)-\mathrm{C}(115)$ & $120.3(3)$ & $\mathrm{C}(114)-\mathrm{C}(115)-\mathrm{C}(116)$ & $118.1(3)$ \\
\hline$C(111)-C(116)-C(115)$ & $122.6(3)$ & $C(122)-C(121)-C(126)$ & $118.3(3)$ \\
\hline $\mathrm{C}(122)-\mathrm{C}(121)-\mathrm{P}(11)$ & $120.7(2)$ & $\mathrm{C}(126)-\mathrm{C}(121)-\mathrm{P}(11)$ & $120.4(2)$ \\
\hline $\mathrm{C}(121)-\mathrm{C}(122)-\mathrm{C}(123)$ & 121.1(3) & $\mathrm{C}(124)-\mathrm{C}(123)-\mathrm{C}(122)$ & $119.9(3)$ \\
\hline
\end{tabular}




\begin{tabular}{|c|c|c|c|}
\hline $\mathrm{C}(125)-\mathrm{C}(124)-\mathrm{C}(123)$ & $118.8(3)$ & $\mathrm{C}(124)-\mathrm{C}(125)-\mathrm{C}(126)$ & $121.3(3)$ \\
\hline$C(125)-C(126)-C(121)$ & $120.5(3)$ & $\mathrm{C}(132)-\mathrm{C}(131)-\mathrm{C}(136)$ & $118.6(3)$ \\
\hline $\mathrm{C}(132)-\mathrm{C}(131)-\mathrm{P}(12)$ & $122.7(2)$ & $\mathrm{C}(136)-\mathrm{C}(131)-\mathrm{P}(12)$ & $118.7(2)$ \\
\hline C(131)-C(132)-C(133) & $120.7(3)$ & $\mathrm{C}(132)-\mathrm{C}(133)-\mathrm{C}(134)$ & $119.8(3)$ \\
\hline $\mathrm{C}(135)-\mathrm{C}(134)-\mathrm{C}(133)$ & $119.9(4)$ & $\mathrm{C}(134)-\mathrm{C}(135)-\mathrm{C}(136)$ & $118.4(3)$ \\
\hline $\mathrm{C}(135)-\mathrm{C}(136)-\mathrm{C}(131)$ & $122.4(3)$ & $\mathrm{C}(142)-\mathrm{C}(141)-\mathrm{C}(146)$ & $119.1(3)$ \\
\hline $\mathrm{C}(142)-\mathrm{C}(141)-\mathrm{P}(12)$ & $119.8(2)$ & $\mathrm{C}(146)-\mathrm{C}(141)-\mathrm{P}(12)$ & $121.1(2)$ \\
\hline $\mathrm{C}(143)-\mathrm{C}(142)-\mathrm{C}(141)$ & $121.7(3)$ & $\mathrm{C}(144)-\mathrm{C}(143)-\mathrm{C}(142)$ & $118.6(3)$ \\
\hline$C(143)-C(144)-C(145)$ & $121.4(3)$ & $\mathrm{C}(146)-\mathrm{C}(145)-\mathrm{C}(144)$ & 121.1(3) \\
\hline$C(145)-C(146)-C(141)$ & 118.1(3) & $\mathrm{C}(152)-\mathrm{C}(151)-\mathrm{C}(156)$ & $118.2(2)$ \\
\hline $\mathrm{C}(152)-\mathrm{C}(151)-\mathrm{P}(13)$ & $122.1(2)$ & $\mathrm{C}(156)-\mathrm{C}(151)-\mathrm{P}(13)$ & $119.7(2)$ \\
\hline $\mathrm{C}(151)-\mathrm{C}(152)-\mathrm{C}(153)$ & $119.7(3)$ & $C(154)-C(153)-C(152)$ & $121.2(3)$ \\
\hline $\mathrm{C}(153)-\mathrm{C}(154)-\mathrm{C}(155)$ & $119.4(3)$ & $\mathrm{C}(156)-\mathrm{C}(155)-\mathrm{C}(154)$ & $120.1(3)$ \\
\hline$C(155)-C(156)-C(151)$ & $121.4(3)$ & $C(162)-C(161)-C(166)$ & $116.5(3)$ \\
\hline $\mathrm{C}(162)-\mathrm{C}(161)-\mathrm{P}(13)$ & $122.3(2)$ & $\mathrm{C}(166)-\mathrm{C}(161)-\mathrm{P}(13)$ & $121.3(2)$ \\
\hline$C(163)-C(162)-C(161)$ & $121.9(3)$ & $\mathrm{C}(162)-\mathrm{C}(163)-\mathrm{C}(164)$ & $120.4(3)$ \\
\hline$C(165)-C(164)-C(163)$ & $118.8(3)$ & $C(164)-C(165)-C(166)$ & $119.8(3)$ \\
\hline$C(165)-C(166)-C(161)$ & $122.6(3)$ & $\mathrm{C}(176)-\mathrm{C}(171)-\mathrm{C}(172)$ & $118.1(3)$ \\
\hline $\mathrm{C}(176)-\mathrm{C}(171)-\mathrm{P}(14)$ & $120.9(2)$ & $\mathrm{C}(172)-\mathrm{C}(171)-\mathrm{P}(14)$ & $121.0(2)$ \\
\hline $\mathrm{C}(173)-\mathrm{C}(172)-\mathrm{C}(171)$ & $120.5(3)$ & $\mathrm{C}(172)-\mathrm{C}(173)-\mathrm{C}(174)$ & $118.1(3)$ \\
\hline $\mathrm{C}(175)-\mathrm{C}(174)-\mathrm{C}(173)$ & $122.6(3)$ & $\mathrm{C}(174)-\mathrm{C}(175)-\mathrm{C}(176)$ & $118.8(3)$ \\
\hline $\mathrm{C}(175)-\mathrm{C}(176)-\mathrm{C}(171)$ & $121.8(3)$ & $\mathrm{C}(186)-\mathrm{C}(181)-\mathrm{C}(182)$ & $116.6(3)$ \\
\hline $\mathrm{C}(186)-\mathrm{C}(181)-\mathrm{P}(14)$ & $122.6(3)$ & $\mathrm{C}(182)-\mathrm{C}(181)-\mathrm{P}(14)$ & $120.1(2)$ \\
\hline $\mathrm{C}(183)-\mathrm{C}(182)-\mathrm{C}(181)$ & $124.0(3)$ & $\mathrm{C}(184)-\mathrm{C}(183)-\mathrm{C}(182)$ & $117.7(3)$ \\
\hline C(185)-C(184)-C(183) & $122.7(4)$ & $\mathrm{C}(184)-\mathrm{C}(185)-\mathrm{C}(186)$ & $119.1(4)$ \\
\hline $\mathrm{C}(181)-\mathrm{C}(186)-\mathrm{C}(185)$ & $119.9(3)$ & $\mathrm{C}(202)-\mathrm{C}(201)-\mathrm{P}(21)$ & $107.56(19)$ \\
\hline $\mathrm{C}(201)-\mathrm{C}(202)-\mathrm{P}(22)$ & $108.65(19)$ & $\mathrm{C}(204)-\mathrm{C}(203)-\mathrm{P}(23)$ & $107.79(19)$ \\
\hline C(203)-C(204)-P(24) & 111.01(19) & $C(216)-C(211)-C(212)$ & $117.5(3)$ \\
\hline $\mathrm{C}(216)-\mathrm{C}(211)-\mathrm{P}(21)$ & $121.8(2)$ & $\mathrm{C}(212)-\mathrm{C}(211)-\mathrm{P}(21)$ & $120.7(2)$ \\
\hline$C(213)-C(212)-C(211)$ & $120.7(3)$ & $\mathrm{C}(212)-\mathrm{C}(213)-\mathrm{C}(214)$ & $120.7(3)$ \\
\hline$C(215)-C(214)-C(213)$ & $119.8(3)$ & $C(216)-C(215)-C(214)$ & $120.6(3)$ \\
\hline$C(215)-C(216)-C(211)$ & $120.7(3)$ & $\mathrm{C}(226)-\mathrm{C}(221)-\mathrm{C}(222)$ & $117.5(3)$ \\
\hline $\mathrm{C}(226)-\mathrm{C}(221)-\mathrm{P}(21)$ & $121.9(2)$ & $\mathrm{C}(222)-\mathrm{C}(221)-\mathrm{P}(21)$ & $120.5(2)$ \\
\hline $\mathrm{C}(221)-\mathrm{C}(222)-\mathrm{C}(223)$ & $120.2(3)$ & $\mathrm{C}(222)-\mathrm{C}(223)-\mathrm{C}(224)$ & $121.1(3)$ \\
\hline $\mathrm{C}(225)-\mathrm{C}(224)-\mathrm{C}(223)$ & $118.2(3)$ & $\mathrm{C}(224)-\mathrm{C}(225)-\mathrm{C}(226)$ & $120.4(4)$ \\
\hline$C(221)-C(226)-C(225)$ & $122.6(3)$ & $\mathrm{C}(236)-\mathrm{C}(231)-\mathrm{C}(232)$ & $118.4(3)$ \\
\hline $\mathrm{C}(236)-\mathrm{C}(231)-\mathrm{P}(22)$ & $121.0(2)$ & $\mathrm{C}(232)-\mathrm{C}(231)-\mathrm{P}(22)$ & $120.6(2)$ \\
\hline$C(233)-C(232)-C(231)$ & $120.6(3)$ & $\mathrm{C}(234)-\mathrm{C}(233)-\mathrm{C}(232)$ & $119.4(3)$ \\
\hline
\end{tabular}




\begin{tabular}{|c|c|c|c|}
\hline$C(233)-C(234)-C(235)$ & $121.9(3)$ & $\mathrm{C}(234)-\mathrm{C}(235)-\mathrm{C}(236)$ & $119.4(3)$ \\
\hline$C(235)-C(236)-C(231)$ & $120.2(3)$ & $\mathrm{C}(242)-\mathrm{C}(241)-\mathrm{C}(246)$ & $116.9(2)$ \\
\hline $\mathrm{C}(242)-\mathrm{C}(241)-\mathrm{P}(22)$ & $121.0(2)$ & $\mathrm{C}(246)-\mathrm{C}(241)-\mathrm{P}(22)$ & $122.1(2)$ \\
\hline$C(243)-C(242)-C(241)$ & $121.2(3)$ & $\mathrm{C}(244)-\mathrm{C}(243)-\mathrm{C}(242)$ & $120.9(3)$ \\
\hline$C(243)-C(244)-C(245)$ & $121.3(3)$ & $\mathrm{C}(244)-\mathrm{C}(245)-\mathrm{C}(246)$ & $118.6(3)$ \\
\hline$C(241)-C(246)-C(245)$ & $121.0(3)$ & $\mathrm{C}(256)-\mathrm{C}(251)-\mathrm{C}(252)$ & $118.4(3)$ \\
\hline $\mathrm{C}(256)-\mathrm{C}(251)-\mathrm{P}(23)$ & $122.3(2)$ & $\mathrm{C}(252)-\mathrm{C}(251)-\mathrm{P}(23)$ & $119.2(2)$ \\
\hline $\mathrm{C}(251)-\mathrm{C}(252)-\mathrm{C}(253)$ & $119.3(3)$ & $\mathrm{C}(254)-\mathrm{C}(253)-\mathrm{C}(252)$ & $122.2(3)$ \\
\hline$C(253)-C(254)-C(255)$ & $118.9(3)$ & $C(254)-C(255)-C(256)$ & $120.2(3)$ \\
\hline$C(251)-C(256)-C(255)$ & $120.9(3)$ & $C(262)-C(261)-C(266)$ & $117.6(2)$ \\
\hline $\mathrm{C}(262)-\mathrm{C}(261)-\mathrm{P}(23)$ & $120.6(2)$ & $\mathrm{C}(266)-\mathrm{C}(261)-\mathrm{P}(23)$ & $121.3(2)$ \\
\hline$C(263)-C(262)-C(261)$ & $121.1(3)$ & $C(264)-C(263)-C(262)$ & $120.9(3)$ \\
\hline$C(263)-C(264)-C(265)$ & $119.7(3)$ & $\mathrm{C}(266)-\mathrm{C}(265)-\mathrm{C}(264)$ & $119.9(3)$ \\
\hline$C(265)-C(266)-C(261)$ & $120.7(3)$ & $\mathrm{C}(276)-\mathrm{C}(271)-\mathrm{C}(272)$ & $119.0(2)$ \\
\hline $\mathrm{C}(276)-\mathrm{C}(271)-\mathrm{P}(24)$ & $121.2(2)$ & $\mathrm{C}(272)-\mathrm{C}(271)-\mathrm{P}(24)$ & $119.8(2)$ \\
\hline $\mathrm{C}(273)-\mathrm{C}(272)-\mathrm{C}(271)$ & $121.1(3)$ & $\mathrm{C}(274)-\mathrm{C}(273)-\mathrm{C}(272)$ & $118.8(3)$ \\
\hline $\mathrm{C}(275)-\mathrm{C}(274)-\mathrm{C}(273)$ & $121.6(3)$ & $\mathrm{C}(274)-\mathrm{C}(275)-\mathrm{C}(276)$ & $119.0(3)$ \\
\hline $\mathrm{C}(271)-\mathrm{C}(276)-\mathrm{C}(275)$ & $120.5(3)$ & $\mathrm{C}(286)-\mathrm{C}(281)-\mathrm{C}(282)$ & $117.0(3)$ \\
\hline $\mathrm{C}(286)-\mathrm{C}(281)-\mathrm{P}(24)$ & $122.8(2)$ & $\mathrm{C}(282)-\mathrm{C}(281)-\mathrm{P}(24)$ & $120.1(2)$ \\
\hline $\mathrm{C}(283)-\mathrm{C}(282)-\mathrm{C}(281)$ & $121.1(3)$ & $\mathrm{C}(284)-\mathrm{C}(283)-\mathrm{C}(282)$ & $120.5(3)$ \\
\hline$C(283)-C(284)-C(285)$ & $118.8(3)$ & $\mathrm{C}(286)-\mathrm{C}(285)-\mathrm{C}(284)$ & $121.0(3)$ \\
\hline $\mathrm{C}(285)-\mathrm{C}(286)-\mathrm{C}(281)$ & $121.5(3)$ & $\mathrm{C}(302)-\mathrm{C}(301)-\mathrm{P}(31)$ & $111.52(18)$ \\
\hline $\mathrm{C}(301)-\mathrm{C}(302)-\mathrm{P}(32)$ & $106.86(17)$ & $\mathrm{C}(304)-\mathrm{C}(303)-\mathrm{P}(33)$ & $112.35(18)$ \\
\hline $\mathrm{C}(303)-\mathrm{C}(304)-\mathrm{P}(34)$ & $108.65(17)$ & $\mathrm{C}(312)-\mathrm{C}(311)-\mathrm{C}(316)$ & $119.4(3)$ \\
\hline $\mathrm{C}(312)-\mathrm{C}(311)-\mathrm{P}(31)$ & $121.2(2)$ & $\mathrm{C}(316)-\mathrm{C}(311)-\mathrm{P}(31)$ & $119.1(2)$ \\
\hline $\mathrm{C}(311)-\mathrm{C}(312)-\mathrm{C}(313)$ & $119.3(3)$ & $\mathrm{C}(314)-\mathrm{C}(313)-\mathrm{C}(312)$ & $120.1(3)$ \\
\hline $\mathrm{C}(313)-\mathrm{C}(314)-\mathrm{C}(315)$ & $121.3(3)$ & $\mathrm{C}(314)-\mathrm{C}(315)-\mathrm{C}(316)$ & $119.1(3)$ \\
\hline $\mathrm{C}(315)-\mathrm{C}(316)-\mathrm{C}(311)$ & $120.9(3)$ & $\mathrm{C}(322)-\mathrm{C}(321)-\mathrm{C}(326)$ & $119.0(3)$ \\
\hline $\mathrm{C}(322)-\mathrm{C}(321)-\mathrm{P}(31)$ & $118.7(2)$ & $\mathrm{C}(326)-\mathrm{C}(321)-\mathrm{P}(31)$ & $122.20(19)$ \\
\hline $\mathrm{C}(321)-\mathrm{C}(322)-\mathrm{C}(323)$ & $119.0(3)$ & $\mathrm{C}(324)-\mathrm{C}(323)-\mathrm{C}(322)$ & $120.7(3)$ \\
\hline $\mathrm{C}(325)-\mathrm{C}(324)-\mathrm{C}(323)$ & $120.1(3)$ & $\mathrm{C}(324)-\mathrm{C}(325)-\mathrm{C}(326)$ & $121.3(3)$ \\
\hline $\mathrm{C}(325)-\mathrm{C}(326)-\mathrm{C}(321)$ & $119.9(2)$ & $\mathrm{C}(336)-\mathrm{C}(331)-\mathrm{C}(332)$ & $118.7(2)$ \\
\hline $\mathrm{C}(336)-\mathrm{C}(331)-\mathrm{P}(32)$ & $121.06(18)$ & $\mathrm{C}(332)-\mathrm{C}(331)-\mathrm{P}(32)$ & $119.90(19)$ \\
\hline $\mathrm{C}(333)-\mathrm{C}(332)-\mathrm{C}(331)$ & $120.9(2)$ & $\mathrm{C}(332)-\mathrm{C}(333)-\mathrm{C}(334)$ & $119.6(2)$ \\
\hline $\mathrm{C}(335)-\mathrm{C}(334)-\mathrm{C}(333)$ & $120.1(3)$ & $\mathrm{C}(334)-\mathrm{C}(335)-\mathrm{C}(336)$ & $120.1(3)$ \\
\hline $\mathrm{C}(331)-\mathrm{C}(336)-\mathrm{C}(335)$ & $120.5(2)$ & $\mathrm{C}(346)-\mathrm{C}(341)-\mathrm{C}(342)$ & $118.4(2)$ \\
\hline $\mathrm{C}(346)-\mathrm{C}(341)-\mathrm{P}(32)$ & $120.2(2)$ & $\mathrm{C}(342)-\mathrm{C}(341)-\mathrm{P}(32)$ & $121.35(19)$ \\
\hline $\mathrm{C}(343)-\mathrm{C}(342)-\mathrm{C}(341)$ & $120.3(2)$ & $\mathrm{C}(344)-\mathrm{C}(343)-\mathrm{C}(342)$ & $121.1(3)$ \\
\hline
\end{tabular}




\begin{tabular}{|c|c|c|c|}
\hline $\mathrm{C}(343)-\mathrm{C}(344)-\mathrm{C}(345)$ & $119.5(3)$ & $\mathrm{C}(346)-\mathrm{C}(345)-\mathrm{C}(344)$ & $119.5(3)$ \\
\hline $\mathrm{C}(345)-\mathrm{C}(346)-\mathrm{C}(341)$ & 121.1(3) & $\mathrm{C}(352)-\mathrm{C}(351)-\mathrm{C}(356)$ & $118.8(3)$ \\
\hline $\mathrm{C}(352)-\mathrm{C}(351)-\mathrm{P}(33)$ & $122.3(2)$ & $\mathrm{C}(356)-\mathrm{C}(351)-\mathrm{P}(33)$ & $118.81(19)$ \\
\hline $\mathrm{C}(351)-\mathrm{C}(352)-\mathrm{C}(353)$ & 121.1(3) & $\mathrm{C}(354)-\mathrm{C}(353)-\mathrm{C}(352)$ & $119.9(3)$ \\
\hline$C(355)-C(354)-C(353)$ & $120.2(3)$ & $C(354)-C(355)-C(356)$ & $119.9(3)$ \\
\hline $\mathrm{C}(351)-\mathrm{C}(356)-\mathrm{C}(355)$ & $119.9(3)$ & $C(366)-C(361)-C(362)$ & $117.7(2)$ \\
\hline $\mathrm{C}(366)-\mathrm{C}(361)-\mathrm{P}(33)$ & $119.5(2)$ & $\mathrm{C}(362)-\mathrm{C}(361)-\mathrm{P}(33)$ & $122.7(2)$ \\
\hline$C(363)-C(362)-C(361)$ & $119.8(3)$ & $\mathrm{C}(362)-\mathrm{C}(363)-\mathrm{C}(364)$ & $122.6(3)$ \\
\hline$C(363)-C(364)-C(365)$ & $118.4(3)$ & $C(366)-C(365)-C(364)$ & $120.4(3)$ \\
\hline$C(365)-C(366)-C(361)$ & 121.1(3) & $\mathrm{C}(372)-\mathrm{C}(371)-\mathrm{C}(376)$ & $118.9(2)$ \\
\hline $\mathrm{C}(372)-\mathrm{C}(371)-\mathrm{P}(34)$ & $119.0(2)$ & $\mathrm{C}(376)-\mathrm{C}(371)-\mathrm{P}(34)$ & $121.6(2)$ \\
\hline $\mathrm{C}(371)-\mathrm{C}(372)-\mathrm{C}(373)$ & $121.4(3)$ & $\mathrm{C}(372)-\mathrm{C}(373)-\mathrm{C}(374)$ & $120.1(3)$ \\
\hline $\mathrm{C}(375)-\mathrm{C}(374)-\mathrm{C}(373)$ & $118.9(3)$ & $\mathrm{C}(374)-\mathrm{C}(375)-\mathrm{C}(376)$ & $120.1(3)$ \\
\hline $\mathrm{C}(371)-\mathrm{C}(376)-\mathrm{C}(375)$ & $120.5(3)$ & $\mathrm{C}(386)-\mathrm{C}(381)-\mathrm{C}(382)$ & $118.9(2)$ \\
\hline $\mathrm{C}(386)-\mathrm{C}(381)-\mathrm{P}(34)$ & $117.9(2)$ & $\mathrm{C}(382)-\mathrm{C}(381)-\mathrm{P}(34)$ & $123.0(2)$ \\
\hline $\mathrm{C}(383)-\mathrm{C}(382)-\mathrm{C}(381)$ & $120.6(3)$ & $\mathrm{C}(384)-\mathrm{C}(383)-\mathrm{C}(382)$ & $118.8(3)$ \\
\hline $\mathrm{C}(385)-\mathrm{C}(384)-\mathrm{C}(383)$ & $121.6(3)$ & $\mathrm{C}(384)-\mathrm{C}(385)-\mathrm{C}(386)$ & $120.3(3)$ \\
\hline $\mathrm{C}(385)-\mathrm{C}(386)-\mathrm{C}(381)$ & $119.6(3)$ & $\mathrm{Cl}(12)-\mathrm{C}(1 \mathrm{~S})-\mathrm{Cl}(11)$ & $110.8(3)$ \\
\hline $\mathrm{Cl}(21)-\mathrm{C}(2 \mathrm{~S})-\mathrm{Cl}(22)$ & $114.4(3)$ & $\mathrm{Cl}(31)-\mathrm{C}(3 \mathrm{~S})-\mathrm{Cl}(32)$ & $117.2(4)$ \\
\hline $\mathrm{Cl}(65)-\mathrm{C}(6 \mathrm{~S})-\mathrm{Cl}(63)$ & $121.4(3)$ & $\mathrm{Cl}(65)-\mathrm{C}(6 \mathrm{~S})-\mathrm{Cl}(61)$ & $97.2(3)$ \\
\hline $\mathrm{Cl}(63)-\mathrm{C}(6 \mathrm{~S})-\mathrm{Cl}(61)$ & $32.6(2)$ & $\mathrm{Cl}(65)-\mathrm{C}(6 \mathrm{~S})-\mathrm{Cl}(62)$ & $28.78(18)$ \\
\hline $\mathrm{Cl}(63)-\mathrm{C}(6 \mathrm{~S})-\mathrm{Cl}(62)$ & $122.5(3)$ & $\mathrm{Cl}(61)-\mathrm{C}(6 \mathrm{~S})-\mathrm{Cl}(62)$ & $112.5(3)$ \\
\hline $\mathrm{Cl}(65)-\mathrm{C}(6 \mathrm{~S})-\mathrm{Cl}(64)$ & $36.3(2)$ & $\mathrm{Cl}(63)-\mathrm{C}(6 \mathrm{~S})-\mathrm{Cl}(64)$ & $99.5(2)$ \\
\hline $\mathrm{Cl}(61)-\mathrm{C}(6 \mathrm{~S})-\mathrm{Cl}(64)$ & $68.2(3)$ & $\mathrm{Cl}(62)-\mathrm{C}(6 \mathrm{~S})-\mathrm{Cl}(64)$ & $64.1(2)$ \\
\hline$C(12 S)-C(11 S)-C(16 S)$ & 120.0 & $\mathrm{C}(13 \mathrm{~S})-\mathrm{C}(12 \mathrm{~S})-\mathrm{C}(11 \mathrm{~S})$ & 120.0 \\
\hline $\mathrm{C}(12 \mathrm{~S})-\mathrm{C}(13 \mathrm{~S})-\mathrm{C}(14 \mathrm{~S})$ & 120.0 & $\mathrm{C}(15 \mathrm{~S})-\mathrm{C}(14 \mathrm{~S})-\mathrm{C}(13 \mathrm{~S})$ & 120.0 \\
\hline$C(14 S)-C(15 S)-C(16 S)$ & 120.0 & $C(15 S)-C(16 S)-C(11 S)$ & 120.0 \\
\hline $\mathrm{Cl}(42)-\mathrm{C}(4 \mathrm{~S})-\mathrm{Cl}(41)$ & $125.2(5)$ & $\mathrm{C}(23 \mathrm{~S})-\mathrm{C}(22 \mathrm{~S})-\mathrm{C}(21 \mathrm{~S})$ & $119.6(7)$ \\
\hline $\mathrm{C}(24 \mathrm{~S})-\mathrm{C}(23 \mathrm{~S})-\mathrm{C}(22 \mathrm{~S})$ & $108.7(6)$ & $\mathrm{C}(23 \mathrm{~S})-\mathrm{C}(24 \mathrm{~S})-\mathrm{C}(25 \mathrm{~S})$ & $97.5(6)$ \\
\hline$C(24 S)-C(25 S)-C(26 S)$ & $108.0(6)$ & $\mathrm{Cl}(51)-\mathrm{C}(5 \mathrm{~S})-\mathrm{Cl}(52)$ & $103.4(4)$ \\
\hline $\mathrm{C}(31 \mathrm{~S})-\mathrm{C}(32 \mathrm{~S})-\mathrm{C}(33 \mathrm{~S})$ & $103.8(6)$ & $\mathrm{C}(34 \mathrm{~S})-\mathrm{C}(33 \mathrm{~S})-\mathrm{C}(32 \mathrm{~S})$ & $108.9(6)$ \\
\hline $\mathrm{C}(33 \mathrm{~S})-\mathrm{C}(34 \mathrm{~S})-\mathrm{C}(35 \mathrm{~S})$ & $105.7(7)$ & $\mathrm{C}(36 \mathrm{~S})-\mathrm{C}(35 \mathrm{~S})-\mathrm{C}(34 \mathrm{~S})$ & $105.9(6)$ \\
\hline
\end{tabular}

Symmetry transformations used to generate equivalent atoms: 
Table S3. Anisotropic displacement parameters $\left(\AA^{2} \times 10^{3}\right)$ for 2,4,6-[4-(Cl(dppe $\left.\left.)_{2} \mathrm{RuC} \equiv \mathrm{C}\right) \mathrm{C}_{6} \mathrm{H}_{4}\right]_{3^{-}}$ 1,3,5- $\mathrm{C}_{3} \mathrm{~N}_{3}(\mathbf{5})$. The anisotropic displacement factor exponent takes the form: $-2 \pi^{2}\left[\mathrm{~h}^{2} \mathrm{a}^{* 2} \mathrm{U}^{11}+\ldots+2\right.$ $\mathrm{hka} \mathrm{k}^{*} \mathrm{U}^{12}$ ]

\begin{tabular}{|c|c|c|c|c|c|c|}
\hline & $\mathrm{U}^{11}$ & $\mathrm{U}^{22}$ & $\mathrm{U}^{33}$ & $\mathrm{U}^{23}$ & $\mathrm{U}^{13}$ & $\mathrm{U}^{12}$ \\
\hline $\mathrm{Ru}(1)$ & $25(1)$ & $18(1)$ & 21(1) & $-1(1)$ & $10(1)$ & $-6(1)$ \\
\hline $\mathrm{Ru}(2)$ & 19(1) & $22(1)$ & 21(1) & $-1(1)$ & $7(1)$ & $-5(1)$ \\
\hline $\mathrm{Ru}(3)$ & $20(1)$ & $16(1)$ & $17(1)$ & $-2(1)$ & $7(1)$ & $-3(1)$ \\
\hline $\mathrm{Cl}(1)$ & $35(1)$ & $24(1)$ & $29(1)$ & $-1(1)$ & $14(1)$ & $-11(1)$ \\
\hline $\mathrm{Cl}(2)$ & $22(1)$ & $30(1)$ & $39(1)$ & 1(1) & $7(1)$ & $-7(1)$ \\
\hline $\mathrm{Cl}(3)$ & $23(1)$ & $22(1)$ & $28(1)$ & $4(1)$ & $8(1)$ & $1(1)$ \\
\hline $\mathrm{P}(11)$ & $30(1)$ & $25(1)$ & $26(1)$ & $-3(1)$ & $14(1)$ & $-6(1)$ \\
\hline $\mathrm{P}(12)$ & $28(1)$ & $28(1)$ & $22(1)$ & $-2(1)$ & $8(1)$ & $-6(1)$ \\
\hline $\mathrm{P}(13)$ & $29(1)$ & $21(1)$ & $23(1)$ & 1(1) & $9(1)$ & $-4(1)$ \\
\hline $\mathrm{P}(14)$ & $24(1)$ & $27(1)$ & $25(1)$ & $-1(1)$ & $7(1)$ & $-7(1)$ \\
\hline $\mathrm{P}(21)$ & $26(1)$ & $25(1)$ & $23(1)$ & $-1(1)$ & $9(1)$ & $-6(1)$ \\
\hline $\mathrm{P}(22)$ & $26(1)$ & $23(1)$ & $26(1)$ & $-1(1)$ & $7(1)$ & $-4(1)$ \\
\hline $\mathrm{P}(23)$ & $23(1)$ & $25(1)$ & $24(1)$ & $0(1)$ & $10(1)$ & $-4(1)$ \\
\hline $\mathrm{P}(24)$ & $22(1)$ & $22(1)$ & $22(1)$ & $-2(1)$ & $5(1)$ & $-5(1)$ \\
\hline $\mathrm{P}(31)$ & $24(1)$ & $20(1)$ & $24(1)$ & $-3(1)$ & $10(1)$ & $-5(1)$ \\
\hline $\mathrm{P}(32)$ & $23(1)$ & $17(1)$ & $21(1)$ & $0(1)$ & $8(1)$ & $-6(1)$ \\
\hline $\mathrm{P}(33)$ & $24(1)$ & $17(1)$ & 19(1) & $1(1)$ & $9(1)$ & $-1(1)$ \\
\hline $\mathrm{P}(34)$ & $20(1)$ & $21(1)$ & 21(1) & $-2(1)$ & $6(1)$ & $-2(1)$ \\
\hline $\mathrm{N}(1)$ & 19(1) & $14(1)$ & 19(1) & $-1(1)$ & $4(1)$ & $-4(1)$ \\
\hline $\mathrm{N}(2)$ & 19(1) & $18(1)$ & $29(1)$ & $3(1)$ & $3(1)$ & $-6(1)$ \\
\hline $\mathrm{N}(3)$ & $17(1)$ & $21(1)$ & $22(1)$ & $3(1)$ & $7(1)$ & $-4(1)$ \\
\hline $\mathrm{C}(11)$ & $31(1)$ & $14(1)$ & $16(1)$ & $-1(1)$ & $18(1)$ & $-1(1)$ \\
\hline $\mathrm{C}(12)$ & $20(1)$ & $13(1)$ & $33(2)$ & $-4(1)$ & $12(1)$ & $-2(1)$ \\
\hline $\mathrm{C}(13)$ & $14(1)$ & $24(1)$ & $27(1)$ & $7(1)$ & $5(1)$ & $-1(1)$ \\
\hline $\mathrm{C}(14)$ & $21(1)$ & $27(1)$ & $24(1)$ & $5(1)$ & $1(1)$ & $-5(1)$ \\
\hline $\mathrm{C}(15)$ & $17(1)$ & $17(1)$ & $30(1)$ & $-1(1)$ & $13(1)$ & $-5(1)$ \\
\hline$C(16)$ & $32(2)$ & $28(2)$ & $25(2)$ & $8(1)$ & $5(1)$ & $-3(1)$ \\
\hline $\mathrm{C}(17)$ & $27(2)$ & $25(2)$ & $27(2)$ & $-2(1)$ & $3(1)$ & $-5(1)$ \\
\hline $\mathrm{C}(18)$ & $28(2)$ & $18(1)$ & $27(2)$ & $-5(1)$ & $7(1)$ & $-1(1)$ \\
\hline $\mathrm{C}(19)$ & $25(1)$ & $19(1)$ & $23(1)$ & $-2(1)$ & $7(1)$ & $2(1)$ \\
\hline $\mathrm{C}(21)$ & $25(1)$ & $19(1)$ & $18(1)$ & $-4(1)$ & $14(1)$ & $2(1)$ \\
\hline $\mathrm{C}(22)$ & $21(1)$ & $25(1)$ & $14(1)$ & $-3(1)$ & $3(1)$ & $-1(1)$ \\
\hline
\end{tabular}




\begin{tabular}{|c|c|c|c|c|c|c|}
\hline$C(23)$ & $31(2)$ & $24(1)$ & $30(2)$ & $2(1)$ & $16(1)$ & $-12(1)$ \\
\hline$C(24)$ & $25(2)$ & $34(2)$ & $25(2)$ & 11(1) & $-3(1)$ & $-2(1)$ \\
\hline$C(25)$ & $22(1)$ & $35(2)$ & $14(1)$ & $-3(1)$ & $9(1)$ & $-10(1)$ \\
\hline$C(26)$ & $34(2)$ & $12(1)$ & $26(1)$ & 1(1) & 11(1) & $-5(1)$ \\
\hline $\mathrm{C}(27)$ & $20(1)$ & $20(1)$ & $26(1)$ & $1(1)$ & $7(1)$ & $-5(1)$ \\
\hline$C(28)$ & $38(2)$ & 19(1) & $20(1)$ & $4(1)$ & $9(1)$ & $0(1)$ \\
\hline$C(29)$ & $16(1)$ & $20(1)$ & $30(1)$ & $2(1)$ & 11(1) & $-2(1)$ \\
\hline$C(31)$ & $19(1)$ & $14(1)$ & 21(1) & $-1(1)$ & $8(1)$ & $2(1)$ \\
\hline$C(32)$ & $27(1)$ & $18(1)$ & $25(1)$ & $2(1)$ & $19(1)$ & $4(1)$ \\
\hline$C(33)$ & $18(1)$ & $29(2)$ & $30(2)$ & $-8(1)$ & $13(1)$ & $-6(1)$ \\
\hline C(34) & $28(1)$ & 21(1) & 21(1) & $5(1)$ & $10(1)$ & $-1(1)$ \\
\hline$C(35)$ & $32(2)$ & $16(1)$ & $20(1)$ & $-5(1)$ & $5(1)$ & $-1(1)$ \\
\hline$C(36)$ & $16(1)$ & $24(1)$ & $19(1)$ & $-7(1)$ & $5(1)$ & $-4(1)$ \\
\hline C(37) & $28(1)$ & $20(1)$ & $28(1)$ & $-2(1)$ & $15(1)$ & $-1(1)$ \\
\hline C(38) & $24(1)$ & $18(1)$ & $24(1)$ & $-5(1)$ & $13(1)$ & $-6(1)$ \\
\hline C(39) & $23(1)$ & $14(1)$ & $19(1)$ & $2(1)$ & $3(1)$ & $-7(1)$ \\
\hline $\mathrm{C}(101)$ & $33(2)$ & $27(2)$ & $35(2)$ & $-1(1)$ & $16(1)$ & $-4(1)$ \\
\hline$C(102)$ & $45(2)$ & $29(2)$ & $21(2)$ & $-4(1)$ & $8(1)$ & $-6(1)$ \\
\hline$C(103)$ & $31(2)$ & $26(2)$ & $24(1)$ & $3(1)$ & $17(1)$ & $0(1)$ \\
\hline$C(104)$ & $34(2)$ & $24(1)$ & $23(1)$ & $4(1)$ & $9(1)$ & $3(1)$ \\
\hline $\mathrm{C}(111)$ & $26(1)$ & $34(2)$ & $29(2)$ & $-5(1)$ & $16(1)$ & $-4(1)$ \\
\hline$C(112)$ & $37(2)$ & $42(2)$ & $45(2)$ & $7(2)$ & $13(2)$ & $-9(2)$ \\
\hline $\mathrm{C}(113)$ & $38(2)$ & $54(2)$ & $71(2)$ & $15(2)$ & $19(2)$ & $-12(2)$ \\
\hline$C(114)$ & $34(2)$ & $54(2)$ & $74(2)$ & $1(2)$ & $20(2)$ & $-7(2)$ \\
\hline$C(115)$ & $34(2)$ & $45(2)$ & $55(2)$ & $3(2)$ & $14(2)$ & $1(2)$ \\
\hline$C(116)$ & $33(2)$ & $38(2)$ & $43(2)$ & $-3(2)$ & $16(1)$ & $-5(2)$ \\
\hline $\mathrm{C}(121)$ & $35(2)$ & $31(2)$ & $18(1)$ & 1(1) & $9(1)$ & $-2(1)$ \\
\hline$C(122)$ & $43(2)$ & $31(2)$ & $38(2)$ & $-6(1)$ & $21(1)$ & $-3(2)$ \\
\hline$C(123)$ & $61(2)$ & $22(2)$ & $42(2)$ & $-11(2)$ & $19(2)$ & $-11(2)$ \\
\hline$C(124)$ & $56(2)$ & $31(2)$ & $43(2)$ & $-7(2)$ & $12(2)$ & $-1(2)$ \\
\hline$C(125)$ & $49(2)$ & $30(2)$ & $40(2)$ & $-1(2)$ & $17(2)$ & $14(2)$ \\
\hline$C(126)$ & $48(2)$ & $28(2)$ & $35(2)$ & $0(1)$ & $15(2)$ & $-4(2)$ \\
\hline$C(131)$ & $33(2)$ & $41(2)$ & $29(2)$ & $10(1)$ & $14(1)$ & $6(1)$ \\
\hline$C(132)$ & $100(2)$ & $70(2)$ & $37(2)$ & $25(2)$ & $40(2)$ & $54(2)$ \\
\hline$C(133)$ & $185(4)$ & $85(3)$ & $48(2)$ & $36(2)$ & $60(2)$ & $75(3)$ \\
\hline$C(134)$ & $133(3)$ & $45(2)$ & $96(3)$ & $27(2)$ & $58(2)$ & $29(2)$ \\
\hline$C(135)$ & $88(3)$ & $46(2)$ & $65(2)$ & $23(2)$ & $26(2)$ & $5(2)$ \\
\hline$C(136)$ & $60(2)$ & $35(2)$ & $40(2)$ & $17(1)$ & $19(2)$ & $1(2)$ \\
\hline
\end{tabular}




\begin{tabular}{|c|c|c|c|c|c|c|}
\hline $\mathrm{C}(141)$ & $44(2)$ & $53(2)$ & 11(1) & $-10(1)$ & $9(1)$ & $-24(2)$ \\
\hline $\mathrm{C}(142)$ & $42(2)$ & $55(2)$ & $20(2)$ & $2(2)$ & $-5(2)$ & $-18(2)$ \\
\hline$C(143)$ & $55(2)$ & $68(2)$ & $31(2)$ & $7(2)$ & $-2(2)$ & $-39(2)$ \\
\hline$C(144)$ & $37(2)$ & $109(3)$ & $35(2)$ & $-7(2)$ & $6(2)$ & $-39(2)$ \\
\hline$C(145)$ & $40(2)$ & $70(2)$ & $41(2)$ & $-13(2)$ & $5(2)$ & $-7(2)$ \\
\hline$C(146)$ & $25(2)$ & $67(2)$ & $41(2)$ & $-4(2)$ & $4(2)$ & $-9(2)$ \\
\hline$C(151)$ & $30(2)$ & $24(1)$ & $24(2)$ & $0(1)$ & $7(1)$ & $-2(1)$ \\
\hline$C(152)$ & $45(2)$ & $24(2)$ & $35(2)$ & $-1(1)$ & $20(1)$ & $-8(1)$ \\
\hline$C(153)$ & $74(2)$ & $23(2)$ & $29(2)$ & $0(1)$ & $19(2)$ & $-9(2)$ \\
\hline$C(154)$ & $69(2)$ & $25(2)$ & $29(2)$ & $-6(1)$ & $17(2)$ & $9(2)$ \\
\hline$C(155)$ & $47(2)$ & $24(2)$ & $31(2)$ & $0(1)$ & 11(1) & $14(1)$ \\
\hline$C(156)$ & $33(2)$ & $27(2)$ & $23(2)$ & $1(1)$ & $5(1)$ & $2(1)$ \\
\hline$C(161)$ & $31(2)$ & $23(1)$ & $22(1)$ & $-6(1)$ & $13(1)$ & $-3(1)$ \\
\hline$C(162)$ & $47(2)$ & $45(2)$ & $23(2)$ & $-1(1)$ & $13(1)$ & $-4(2)$ \\
\hline$C(163)$ & $38(2)$ & $65(2)$ & $38(2)$ & $11(2)$ & $2(2)$ & $12(2)$ \\
\hline$C(164)$ & $29(2)$ & $70(2)$ & $47(2)$ & $1(2)$ & $10(2)$ & $-3(2)$ \\
\hline$C(165)$ & $31(2)$ & $48(2)$ & $38(2)$ & $-8(2)$ & $19(1)$ & $-11(2)$ \\
\hline$C(166)$ & $27(2)$ & $39(2)$ & $35(2)$ & $1(1)$ & $10(1)$ & $-7(1)$ \\
\hline$C(171)$ & $27(2)$ & $35(2)$ & $36(2)$ & $2(1)$ & $10(1)$ & $5(1)$ \\
\hline $\mathrm{C}(172)$ & $45(2)$ & $53(2)$ & $42(2)$ & $2(2)$ & $-1(2)$ & $14(2)$ \\
\hline$C(173)$ & $74(2)$ & $80(2)$ & $35(2)$ & $23(2)$ & $6(2)$ & $37(2)$ \\
\hline $\mathrm{C}(174)$ & $75(2)$ & $33(2)$ & $69(2)$ & $13(2)$ & $6(2)$ & $20(2)$ \\
\hline$C(175)$ & $38(2)$ & $39(2)$ & $48(2)$ & $5(2)$ & $6(2)$ & $5(2)$ \\
\hline$C(176)$ & $24(1)$ & $31(2)$ & $33(2)$ & $2(1)$ & $14(1)$ & $4(1)$ \\
\hline $\mathrm{C}(181)$ & $28(2)$ & $52(2)$ & $24(2)$ & $-2(1)$ & $9(1)$ & $-16(2)$ \\
\hline C(182) & $56(2)$ & $44(2)$ & $47(2)$ & $8(2)$ & $6(2)$ & $-28(2)$ \\
\hline $\mathrm{C}(183)$ & $67(2)$ & $51(2)$ & $43(2)$ & $5(2)$ & $2(2)$ & $-19(2)$ \\
\hline$C(184)$ & $94(3)$ & $43(2)$ & $49(2)$ & $-10(2)$ & $-3(2)$ & $-29(2)$ \\
\hline$C(185)$ & $44(2)$ & $91(3)$ & 101(3) & $19(2)$ & $28(2)$ & $-11(2)$ \\
\hline$C(186)$ & $53(2)$ & $64(2)$ & $64(3)$ & $-28(2)$ & $4(2)$ & $-21(2)$ \\
\hline$C(201)$ & $27(2)$ & $31(2)$ & $25(2)$ & $5(1)$ & $3(1)$ & $-2(1)$ \\
\hline$C(202)$ & $33(2)$ & $24(2)$ & $34(2)$ & $1(1)$ & $14(1)$ & $-6(1)$ \\
\hline$C(203)$ & $26(1)$ & $31(2)$ & 18(1) & $3(1)$ & $6(1)$ & $-8(1)$ \\
\hline$C(204)$ & $25(1)$ & $24(1)$ & $25(1)$ & $4(1)$ & $11(1)$ & $-2(1)$ \\
\hline $\mathrm{C}(211)$ & $39(2)$ & $36(2)$ & $31(2)$ & $5(1)$ & $24(1)$ & $-1(1)$ \\
\hline $\mathrm{C}(212)$ & $44(2)$ & $41(2)$ & $41(2)$ & $-9(2)$ & $24(1)$ & $-12(2)$ \\
\hline $\mathrm{C}(213)$ & $55(2)$ & $44(2)$ & $66(2)$ & $-5(2)$ & $37(2)$ & $10(2)$ \\
\hline$C(214)$ & $51(2)$ & $72(2)$ & $63(2)$ & $9(2)$ & $44(2)$ & $16(2)$ \\
\hline
\end{tabular}




\begin{tabular}{|c|c|c|c|c|c|c|}
\hline $\mathrm{C}(215)$ & $35(2)$ & $59(2)$ & $40(2)$ & $15(2)$ & $8(2)$ & $-2(2)$ \\
\hline$C(216)$ & $39(2)$ & $29(2)$ & $38(2)$ & $8(1)$ & $22(1)$ & $-1(1)$ \\
\hline $\mathrm{C}(221)$ & $36(2)$ & $42(2)$ & $17(1)$ & $0(1)$ & $6(1)$ & $-6(2)$ \\
\hline $\mathrm{C}(222)$ & $48(2)$ & $33(2)$ & $26(2)$ & $1(1)$ & $3(2)$ & $-16(2)$ \\
\hline $\mathrm{C}(223)$ & $69(2)$ & $63(2)$ & $31(2)$ & $3(2)$ & $-12(2)$ & $-38(2)$ \\
\hline $\mathrm{C}(224)$ & 91(3) & $79(3)$ & $27(2)$ & $3(2)$ & $-13(2)$ & $-21(2)$ \\
\hline$C(225)$ & $129(4)$ & $80(3)$ & $27(2)$ & $15(2)$ & $4(2)$ & $-34(3)$ \\
\hline$C(226)$ & 107(3) & $64(2)$ & $31(2)$ & $0(2)$ & $5(2)$ & $-57(2)$ \\
\hline$C(231)$ & $31(2)$ & $25(2)$ & $41(2)$ & $9(1)$ & $5(1)$ & $5(1)$ \\
\hline C(232) & $30(2)$ & $32(2)$ & $49(2)$ & $8(1)$ & 11(1) & $-4(1)$ \\
\hline $\mathrm{C}(233)$ & $30(2)$ & $42(2)$ & $61(2)$ & $9(2)$ & $9(2)$ & $6(2)$ \\
\hline$C(234)$ & $46(2)$ & $44(2)$ & $75(2)$ & $10(2)$ & $34(2)$ & $13(2)$ \\
\hline$C(235)$ & $51(2)$ & $44(2)$ & $59(2)$ & $5(2)$ & $37(2)$ & $15(2)$ \\
\hline$C(236)$ & $47(2)$ & $30(2)$ & $38(2)$ & $5(1)$ & $17(1)$ & $-7(2)$ \\
\hline $\mathrm{C}(241)$ & $28(2)$ & $23(2)$ & $34(2)$ & $-3(1)$ & $4(1)$ & $-2(1)$ \\
\hline $\mathrm{C}(242)$ & $26(1)$ & $29(2)$ & $31(2)$ & $7(1)$ & $12(1)$ & $-1(1)$ \\
\hline$C(243)$ & $36(2)$ & $38(2)$ & $31(2)$ & $-5(2)$ & $3(2)$ & $-8(2)$ \\
\hline$C(244)$ & $58(2)$ & $39(2)$ & $54(2)$ & $-9(2)$ & $-4(2)$ & $-23(2)$ \\
\hline$C(245)$ & $82(3)$ & $32(2)$ & $79(3)$ & $11(2)$ & $-19(2)$ & $-22(2)$ \\
\hline$C(246)$ & $66(2)$ & $32(2)$ & $58(2)$ & $17(2)$ & $-9(2)$ & $4(2)$ \\
\hline$C(251)$ & $35(2)$ & $26(2)$ & $26(1)$ & $6(1)$ & $17(1)$ & $1(1)$ \\
\hline$C(252)$ & $20(1)$ & $38(2)$ & $50(2)$ & 11(1) & $14(1)$ & $-2(1)$ \\
\hline $\mathrm{C}(253)$ & $34(2)$ & $33(2)$ & $57(2)$ & $13(2)$ & $21(1)$ & $-1(1)$ \\
\hline$C(254)$ & $49(2)$ & $50(2)$ & $39(2)$ & $13(2)$ & $28(1)$ & $21(2)$ \\
\hline$C(255)$ & $41(2)$ & $43(2)$ & $27(2)$ & $3(1)$ & $13(1)$ & $6(2)$ \\
\hline$C(256)$ & $32(2)$ & $39(2)$ & $31(2)$ & $2(1)$ & $13(1)$ & $-2(1)$ \\
\hline$C(261)$ & $25(1)$ & $23(1)$ & $23(1)$ & $-3(1)$ & $7(1)$ & $-11(1)$ \\
\hline$C(262)$ & $36(2)$ & $28(2)$ & $33(2)$ & $-3(1)$ & $17(1)$ & $-11(1)$ \\
\hline$C(263)$ & $42(2)$ & $39(2)$ & $22(2)$ & $-3(1)$ & $5(1)$ & $-2(2)$ \\
\hline$C(264)$ & $36(2)$ & $36(2)$ & $29(2)$ & $-5(1)$ & $6(1)$ & $-7(2)$ \\
\hline$C(265)$ & $27(2)$ & $44(2)$ & $33(2)$ & $-3(2)$ & $2(1)$ & $-9(2)$ \\
\hline$C(266)$ & $39(2)$ & $34(2)$ & $28(2)$ & $3(1)$ & $15(1)$ & $-2(1)$ \\
\hline $\mathrm{C}(271)$ & $28(2)$ & $23(1)$ & $25(2)$ & $1(1)$ & $8(1)$ & $-1(1)$ \\
\hline$C(272)$ & $33(2)$ & $26(2)$ & $30(2)$ & $4(1)$ & $9(1)$ & $-3(1)$ \\
\hline$C(273)$ & $35(2)$ & $33(2)$ & $25(2)$ & $-1(1)$ & $2(1)$ & $-9(1)$ \\
\hline$C(274)$ & $45(2)$ & $38(2)$ & $39(2)$ & $-16(2)$ & $2(2)$ & $-13(2)$ \\
\hline$C(275)$ & $51(2)$ & $48(2)$ & $47(2)$ & $-9(2)$ & $18(2)$ & $-15(2)$ \\
\hline $\mathrm{C}(276)$ & $29(2)$ & $37(2)$ & $28(2)$ & $-10(1)$ & $3(1)$ & $-12(1)$ \\
\hline
\end{tabular}




\begin{tabular}{|c|c|c|c|c|c|c|}
\hline$C(281)$ & $31(2)$ & $26(2)$ & $28(2)$ & $0(1)$ & $9(1)$ & $-4(1)$ \\
\hline$C(282)$ & $27(2)$ & $20(2)$ & $46(2)$ & $-3(1)$ & $5(1)$ & $-5(1)$ \\
\hline$C(283)$ & $32(2)$ & $35(2)$ & $43(2)$ & $-5(2)$ & $4(2)$ & $-7(2)$ \\
\hline$C(284)$ & $29(2)$ & $55(2)$ & $39(2)$ & $-2(2)$ & $1(2)$ & $14(2)$ \\
\hline$C(285)$ & $45(2)$ & $55(2)$ & $52(2)$ & $29(2)$ & $10(2)$ & $13(2)$ \\
\hline$C(286)$ & $35(2)$ & $44(2)$ & $47(2)$ & 21(1) & $16(1)$ & $6(2)$ \\
\hline$C(301)$ & $29(1)$ & $28(2)$ & $18(1)$ & $-4(1)$ & 11(1) & $-6(1)$ \\
\hline$C(302)$ & $39(2)$ & $19(1)$ & $13(1)$ & $-2(1)$ & $3(1)$ & $-12(1)$ \\
\hline$C(303)$ & $28(1)$ & $17(1)$ & $12(1)$ & $-5(1)$ & $9(1)$ & $-4(1)$ \\
\hline$C(304)$ & $38(2)$ & $20(1)$ & $22(1)$ & $0(1)$ & $14(1)$ & $-1(1)$ \\
\hline$C(311)$ & $30(2)$ & $19(1)$ & $25(2)$ & $-11(1)$ & $6(1)$ & $-8(1)$ \\
\hline$C(312)$ & $17(1)$ & $32(2)$ & $55(2)$ & $-8(2)$ & $18(1)$ & $-6(1)$ \\
\hline$C(313)$ & $45(2)$ & $36(2)$ & $50(2)$ & $-2(2)$ & $14(2)$ & $8(2)$ \\
\hline$C(314)$ & $46(2)$ & $42(2)$ & $79(2)$ & $-6(2)$ & $29(2)$ & $9(2)$ \\
\hline$C(315)$ & $53(2)$ & $57(2)$ & $63(2)$ & $6(2)$ & $39(2)$ & $16(2)$ \\
\hline$C(316)$ & $38(2)$ & $56(2)$ & $45(2)$ & $4(2)$ & $21(2)$ & $-2(2)$ \\
\hline$C(321)$ & $30(1)$ & $24(2)$ & $32(2)$ & $-3(1)$ & $23(1)$ & $-3(1)$ \\
\hline$C(322)$ & $37(2)$ & $32(2)$ & $31(2)$ & $-11(1)$ & $20(1)$ & $-12(1)$ \\
\hline$C(323)$ & $52(2)$ & $34(2)$ & $49(2)$ & $-15(2)$ & $34(1)$ & $-20(2)$ \\
\hline$C(324)$ & $71(2)$ & $27(2)$ & $66(2)$ & $10(1)$ & $52(2)$ & $-5(2)$ \\
\hline$C(325)$ & $53(2)$ & $41(2)$ & $63(2)$ & $25(1)$ & $43(1)$ & $14(2)$ \\
\hline$C(326)$ & $35(2)$ & $30(2)$ & $37(2)$ & $5(1)$ & $23(1)$ & $4(1)$ \\
\hline$C(331)$ & $28(1)$ & $14(1)$ & $25(1)$ & $-1(1)$ & $12(1)$ & $-6(1)$ \\
\hline$C(332)$ & $34(2)$ & $22(1)$ & $23(1)$ & $3(1)$ & $14(1)$ & $-10(1)$ \\
\hline$C(333)$ & $38(2)$ & $24(2)$ & $27(2)$ & $-3(1)$ & $14(1)$ & $-11(1)$ \\
\hline$C(334)$ & $36(2)$ & $18(2)$ & $58(2)$ & $-11(1)$ & $29(1)$ & $-8(1)$ \\
\hline$C(335)$ & $24(1)$ & $27(2)$ & $53(2)$ & $5(1)$ & $18(1)$ & $6(1)$ \\
\hline$C(336)$ & $28(1)$ & $18(1)$ & $47(2)$ & $-3(1)$ & $20(1)$ & $-2(1)$ \\
\hline$C(341)$ & 19(1) & $20(1)$ & $16(1)$ & $-5(1)$ & $6(1)$ & $-5(1)$ \\
\hline$C(342)$ & $27(2)$ & $27(2)$ & $17(1)$ & $-2(1)$ & $6(1)$ & $-5(1)$ \\
\hline$C(343)$ & $35(2)$ & $24(2)$ & $23(1)$ & $-4(1)$ & $18(1)$ & $-5(1)$ \\
\hline$C(344)$ & $16(1)$ & $34(2)$ & $38(2)$ & $1(1)$ & $4(1)$ & $-1(1)$ \\
\hline$C(345)$ & $33(2)$ & $22(2)$ & $32(2)$ & $1(1)$ & $-4(1)$ & $0(1)$ \\
\hline$C(346)$ & $27(1)$ & $10(1)$ & $29(2)$ & $-6(1)$ & $4(1)$ & $-8(1)$ \\
\hline$C(351)$ & $17(1)$ & $16(1)$ & $26(1)$ & $2(1)$ & $9(1)$ & $-2(1)$ \\
\hline$C(352)$ & $20(1)$ & $20(1)$ & 19(1) & $-2(1)$ & $6(1)$ & $-3(1)$ \\
\hline$C(353)$ & $33(2)$ & 19(1) & $24(1)$ & $8(1)$ & $7(1)$ & $-1(1)$ \\
\hline$C(354)$ & $37(2)$ & $32(2)$ & $27(2)$ & $3(1)$ & $11(1)$ & $-5(1)$ \\
\hline
\end{tabular}




\begin{tabular}{|c|c|c|c|c|c|c|}
\hline$C(355)$ & $38(2)$ & $32(2)$ & $30(2)$ & $2(1)$ & $19(1)$ & $-2(1)$ \\
\hline$C(356)$ & $29(2)$ & $25(2)$ & $28(2)$ & $-1(1)$ & $7(1)$ & $-7(1)$ \\
\hline $\mathrm{C}(361)$ & $25(1)$ & $26(1)$ & $15(1)$ & $-5(1)$ & $7(1)$ & $5(1)$ \\
\hline$C(362)$ & $26(2)$ & $32(2)$ & $31(2)$ & $-4(1)$ & $10(1)$ & $4(1)$ \\
\hline C(363) & $26(2)$ & $38(2)$ & $38(2)$ & $-6(2)$ & $3(1)$ & $1(1)$ \\
\hline C(364) & $33(2)$ & $46(2)$ & $38(2)$ & $-6(2)$ & $6(2)$ & $15(2)$ \\
\hline$C(365)$ & $67(2)$ & $33(2)$ & $22(1)$ & $2(1)$ & $27(1)$ & $14(2)$ \\
\hline$C(366)$ & $35(2)$ & $27(2)$ & $22(1)$ & $3(1)$ & $13(1)$ & $5(1)$ \\
\hline $\mathrm{C}(371)$ & $22(1)$ & $15(1)$ & $26(2)$ & $-5(1)$ & $2(1)$ & $-5(1)$ \\
\hline C(372) & $23(1)$ & $24(1)$ & $23(1)$ & $-4(1)$ & $4(1)$ & $-8(1)$ \\
\hline C(373) & $38(2)$ & $29(2)$ & $24(2)$ & $-2(1)$ & $4(1)$ & $-8(1)$ \\
\hline $\mathrm{C}(374)$ & $33(2)$ & $24(2)$ & $38(2)$ & $9(1)$ & $-1(1)$ & $-1(1)$ \\
\hline$C(375)$ & $25(2)$ & $26(2)$ & $28(2)$ & $-10(1)$ & $1(1)$ & $-8(1)$ \\
\hline C(376) & $25(1)$ & $22(2)$ & $32(2)$ & $-5(1)$ & $12(1)$ & $-7(1)$ \\
\hline C(381) & $19(1)$ & $27(2)$ & $23(2)$ & $-5(1)$ & $3(1)$ & $-8(1)$ \\
\hline C(382) & $35(2)$ & $24(2)$ & $17(1)$ & $-1(1)$ & $8(1)$ & $-3(1)$ \\
\hline C(383) & $27(2)$ & $29(2)$ & $45(2)$ & $-3(1)$ & $13(1)$ & $-10(1)$ \\
\hline$C(384)$ & $20(2)$ & $41(2)$ & $50(2)$ & $-4(2)$ & $9(1)$ & $-2(1)$ \\
\hline$C(385)$ & $33(2)$ & $23(2)$ & $49(2)$ & $-3(2)$ & $6(2)$ & $-2(1)$ \\
\hline C(386) & $26(2)$ & $21(2)$ & $35(2)$ & $-7(1)$ & $4(1)$ & $-6(1)$ \\
\hline $\mathrm{Cl}(11)$ & $118(1)$ & 109(1) & $96(1)$ & $8(1)$ & $47(1)$ & $-15(1)$ \\
\hline $\mathrm{Cl}(12)$ & $91(1)$ & 171(1) & $89(1)$ & $-9(1)$ & $40(1)$ & $15(1)$ \\
\hline$C(2 S)$ & 103(3) & $81(3)$ & $91(3)$ & $-9(3)$ & $19(3)$ & $-9(3)$ \\
\hline $\mathrm{Cl}(21)$ & 191(2) & $110(1)$ & $91(1)$ & $8(1)$ & $0(1)$ & $39(1)$ \\
\hline $\mathrm{Cl}(22)$ & 131(1) & 116(1) & 109(1) & $29(1)$ & $15(1)$ & $29(1)$ \\
\hline $\mathrm{C}(3 \mathrm{~S})$ & $65(4)$ & 182(7) & $370(11)$ & $-6(7)$ & $16(6)$ & $-20(4)$ \\
\hline $\mathrm{Cl}(31)$ & $209(2)$ & $228(2)$ & $153(1)$ & $-33(2)$ & $90(1)$ & $-8(2)$ \\
\hline $\mathrm{Cl}(32)$ & $246(2)$ & $234(2)$ & 131(1) & $-12(1)$ & $67(1)$ & $-94(2)$ \\
\hline $\mathrm{C}(11 \mathrm{~S})$ & 137(4) & 109(4) & $92(4)$ & $19(3)$ & $-34(3)$ & $31(3)$ \\
\hline $\mathrm{C}(12 \mathrm{~S})$ & $357(6)$ & $82(3)$ & $142(4)$ & $-64(3)$ & $-199(3)$ & $120(3)$ \\
\hline$C(13 S)$ & 201(6) & $72(4)$ & $163(6)$ & $-32(4)$ & $-8(5)$ & $16(4)$ \\
\hline $\mathrm{C}(14 \mathrm{~S})$ & $143(5)$ & 133(4) & $73(3)$ & $-44(3)$ & $-37(3)$ & $36(4)$ \\
\hline$C(15 S)$ & $154(5)$ & 194(6) & $69(4)$ & $-41(4)$ & $-30(4)$ & $71(4)$ \\
\hline$C(16 S)$ & $146(4)$ & $184(5)$ & $86(4)$ & $-29(4)$ & $-20(4)$ & $98(4)$ \\
\hline$C(4 S)$ & $116(4)$ & $219(6)$ & $57(5)$ & $-2(5)$ & $-9(4)$ & $152(3)$ \\
\hline $\mathrm{Cl}(41)$ & 161(2) & 121(2) & 149(2) & $42(2)$ & $71(2)$ & $56(2)$ \\
\hline $\mathrm{Cl}(42)$ & $215(3)$ & $184(2)$ & $80(2)$ & $-23(2)$ & $31(2)$ & $84(2)$ \\
\hline $\mathrm{C}(5 \mathrm{~S})$ & $48(5)$ & $48(5)$ & $48(5)$ & $6(4)$ & $11(4)$ & $2(4)$ \\
\hline
\end{tabular}




$\begin{array}{lllllll}\mathrm{Cl}(51) & 129(2) & 47(1) & 55(2) & -7(1) & 16(2) & 29(2) \\ \mathrm{Cl}(52) & 23(1) & 69(2) & 113(2) & 27(1) & -17(1) & -11(1)\end{array}$


Table S4. Hydrogen coordinates ( $\left.\times 10^{4}\right)$ and isotropic displacement parameters $\left(\AA^{2} \times 10^{3}\right)$ for 2,4,6-[4-(Cl(dppe $\left.\left.)_{2} \mathrm{RuC} \equiv \mathrm{C}\right) \mathrm{C}_{6} \mathrm{H}_{4}\right]_{3}-1,3,5-\mathrm{C}_{3} \mathrm{~N}_{3}(\mathbf{5})$.

\begin{tabular}{|c|c|c|c|c|}
\hline & $\mathrm{x}$ & $\mathrm{y}$ & $\mathrm{z}$ & $\mathrm{U}(\mathrm{eq})$ \\
\hline $\mathrm{H}(13 \mathrm{~A})$ & 5984 & 1931 & 3193 & 25 \\
\hline $\mathrm{H}(14 \mathrm{~A})$ & 6497 & 2928 & 2947 & 30 \\
\hline $\mathrm{H}(16 \mathrm{~A})$ & 4747 & 2854 & 1756 & 34 \\
\hline $\mathrm{H}(17 \mathrm{~A})$ & 4224 & 1857 & 2001 & 33 \\
\hline $\mathrm{H}(23 \mathrm{~A})$ & 2605 & 1187 & 2180 & 33 \\
\hline $\mathrm{H}(24 \mathrm{~A})$ & 1328 & 897 & 1856 & 35 \\
\hline $\mathrm{H}(26 \mathrm{~A})$ & 1615 & -1081 & 2327 & 28 \\
\hline $\mathrm{H}(27 \mathrm{~A})$ & 2863 & -757 & 2688 & 27 \\
\hline $\mathrm{H}(33 \mathrm{~A})$ & 4243 & -790 & 3682 & 31 \\
\hline $\mathrm{H}(34 \mathrm{~A})$ & 4860 & -1482 & 4267 & 26 \\
\hline $\mathrm{H}(36 \mathrm{~A})$ & 6822 & -386 & 4302 & 24 \\
\hline $\mathrm{H}(37 \mathrm{~A})$ & 6177 & 318 & 3727 & 29 \\
\hline $\mathrm{H}(10 \mathrm{~A})$ & 7338 & 5357 & 868 & 37 \\
\hline $\mathrm{H}(10 \mathrm{~B})$ & 7456 & 4573 & 593 & 37 \\
\hline $\mathrm{H}(10 \mathrm{C})$ & 6222 & 4173 & 614 & 39 \\
\hline $\mathrm{H}(10 \mathrm{D})$ & 6165 & 4897 & 349 & 39 \\
\hline $\mathrm{H}(10 \mathrm{E})$ & 5837 & 5437 & 3210 & 31 \\
\hline $\mathrm{H}(10 \mathrm{~F})$ & 5968 & 4674 & 2911 & 31 \\
\hline $\mathrm{H}(10 \mathrm{G})$ & 7100 & 5022 & 3475 & 32 \\
\hline $\mathrm{H}(10 \mathrm{H})$ & 7080 & 5823 & 3315 & 32 \\
\hline $\mathrm{H}(11 \mathrm{~A})$ & 8554 & 5547 & 1205 & 49 \\
\hline $\mathrm{H}(11 \mathrm{~B})$ & 9874 & 5687 & 1372 & 63 \\
\hline $\mathrm{H}(11 \mathrm{C})$ & 10634 & 4975 & 1880 & 64 \\
\hline H(11D) & 10104 & 4015 & 2179 & 53 \\
\hline $\mathrm{H}(11 \mathrm{E})$ & 8789 & 3877 & 2020 & 45 \\
\hline $\mathrm{H}(12 \mathrm{~A})$ & 6481 & 3286 & 1386 & 44 \\
\hline $\mathrm{H}(12 \mathrm{~B})$ & 6305 & 2096 & 981 & 50 \\
\hline $\mathrm{H}(12 \mathrm{C})$ & 7197 & 1612 & 556 & 53 \\
\hline $\mathrm{H}(12 \mathrm{D})$ & 8256 & 2318 & 562 & 47 \\
\hline $\mathrm{H}(12 \mathrm{E})$ & 8417 & 3509 & 946 & 44 \\
\hline $\mathrm{H}(13 \mathrm{~B})$ & 5552 & 5781 & 221 & 76 \\
\hline $\mathrm{H}(13 \mathrm{C})$ & 5370 & 6950 & 13 & 117 \\
\hline
\end{tabular}




\begin{tabular}{|c|c|c|c|c|}
\hline $\mathrm{H}(13 \mathrm{D})$ & 5616 & 7945 & 641 & 101 \\
\hline $\mathrm{H}(13 \mathrm{E})$ & 5786 & 7743 & 1465 & 76 \\
\hline $\mathrm{H}(13 \mathrm{~F})$ & 5944 & 6565 & 1657 & 51 \\
\hline $\mathrm{H}(14 \mathrm{~B})$ & 5318 & 3708 & 1113 & 49 \\
\hline $\mathrm{H}(14 \mathrm{C})$ & 4134 & 3119 & 894 & 64 \\
\hline $\mathrm{H}(14 \mathrm{D})$ & 3089 & 3775 & 631 & 75 \\
\hline $\mathrm{H}(14 \mathrm{E})$ & 3200 & 5008 & 587 & 64 \\
\hline $\mathrm{H}(14 \mathrm{~F})$ & 4378 & 5620 & 803 & 55 \\
\hline $\mathrm{H}(15 \mathrm{~A})$ & 6845 & 6903 & 2399 & 40 \\
\hline $\mathrm{H}(15 \mathrm{~B})$ & 6922 & 8104 & 2794 & 50 \\
\hline $\mathrm{H}(15 \mathrm{C})$ & 6062 & 8500 & 3253 & 49 \\
\hline $\mathrm{H}(15 \mathrm{D})$ & 5131 & 7682 & 3350 & 40 \\
\hline $\mathrm{H}(15 \mathrm{E})$ & 5087 & 6485 & 3006 & 34 \\
\hline $\mathrm{H}(16 \mathrm{~B})$ & 4661 & 6453 & 1961 & 46 \\
\hline $\mathrm{H}(16 \mathrm{C})$ & 3375 & 6281 & 1716 & 57 \\
\hline$H(16 D)$ & 2794 & 5157 & 1754 & 59 \\
\hline $\mathrm{H}(16 \mathrm{E})$ & 3540 & 4223 & 2036 & 46 \\
\hline $\mathrm{H}(16 \mathrm{~F})$ & 4828 & 4412 & 2290 & 41 \\
\hline $\mathrm{H}(17 \mathrm{~B})$ & 8144 & 4446 & 3651 & 58 \\
\hline $\mathrm{H}(17 \mathrm{C})$ & 8497 & 3302 & 3870 & 75 \\
\hline $\mathrm{H}(17 \mathrm{D})$ & 8426 & 2333 & 3239 & 72 \\
\hline $\mathrm{H}(17 \mathrm{E})$ & 8023 & 2477 & 2421 & 51 \\
\hline $\mathrm{H}(17 \mathrm{~F})$ & 7587 & 3588 & 2207 & 34 \\
\hline $\mathrm{H}(18 \mathrm{~A})$ & 7786 & 6530 & 3049 & 60 \\
\hline $\mathrm{H}(18 \mathrm{~B})$ & 8807 & 7351 & 3252 & 67 \\
\hline $\mathrm{H}(18 \mathrm{C})$ & 9985 & 6932 & 3236 & 80 \\
\hline $\mathrm{H}(18 \mathrm{D})$ & 10161 & 5742 & 3007 & 92 \\
\hline $\mathrm{H}(18 \mathrm{E})$ & 9105 & 4868 & 2837 & 78 \\
\hline $\mathrm{H}(20 \mathrm{~A})$ & -335 & 371 & 861 & 34 \\
\hline $\mathrm{H}(20 \mathrm{~B})$ & -796 & 246 & 292 & 34 \\
\hline $\mathrm{H}(20 \mathrm{C})$ & -1957 & 255 & 545 & 35 \\
\hline $\mathrm{H}(20 \mathrm{D})$ & -1480 & 996 & 786 & 35 \\
\hline $\mathrm{H}(20 \mathrm{E})$ & -1878 & -2142 & 2445 & 30 \\
\hline $\mathrm{H}(20 \mathrm{~F})$ & -2229 & -2103 & 1866 & 30 \\
\hline $\mathrm{H}(20 \mathrm{G})$ & -1390 & -3016 & 1884 & 28 \\
\hline $\mathrm{H}(20 \mathrm{H})$ & -757 & -2539 & 2297 & 28 \\
\hline $\mathrm{H}(21 \mathrm{~A})$ & -366 & -2051 & 60 & 49 \\
\hline $\mathrm{H}(21 \mathrm{~B})$ & 770 & -2436 & -69 & 63 \\
\hline
\end{tabular}




\begin{tabular}{|c|c|c|c|c|}
\hline $\mathrm{H}(21 \mathrm{C})$ & 1884 & -1761 & 315 & 68 \\
\hline $\mathrm{H}(21 \mathrm{D})$ & 1842 & -662 & 800 & 53 \\
\hline $\mathrm{H}(21 \mathrm{E})$ & 708 & -240 & 920 & 40 \\
\hline $\mathrm{H}(22 \mathrm{~A})$ & -1961 & -2013 & 317 & 44 \\
\hline $\mathrm{H}(22 \mathrm{~B})$ & -2758 & -2467 & -436 & 71 \\
\hline $\mathrm{H}(22 \mathrm{C})$ & -2789 & -1903 & -1153 & 85 \\
\hline $\mathrm{H}(22 \mathrm{D})$ & -2022 & -892 & -1090 & 97 \\
\hline $\mathrm{H}(22 \mathrm{E})$ & -1202 & -463 & -343 & 84 \\
\hline $\mathrm{H}(23 \mathrm{~B})$ & -3037 & -46 & 835 & 43 \\
\hline $\mathrm{H}(23 \mathrm{C})$ & -4213 & 344 & 916 & 53 \\
\hline $\mathrm{H}(23 \mathrm{D})$ & -4308 & 1145 & 1597 & 62 \\
\hline $\mathrm{H}(23 \mathrm{E})$ & -3260 & 1546 & 2218 & 57 \\
\hline $\mathrm{H}(23 \mathrm{~F})$ & -2063 & 1187 & 2140 & 45 \\
\hline $\mathrm{H}(24 \mathrm{~B})$ & -468 & 338 & 2375 & 33 \\
\hline $\mathrm{H}(24 \mathrm{C})$ & 279 & 1243 & 2914 & 44 \\
\hline $\mathrm{H}(24 \mathrm{D})$ & 371 & 2383 & 2701 & 66 \\
\hline $\mathrm{H}(24 \mathrm{E})$ & -317 & 2677 & 1952 & 85 \\
\hline $\mathrm{H}(24 \mathrm{~F})$ & -1067 & 1762 & 1383 & 66 \\
\hline $\mathrm{H}(25 \mathrm{~A})$ & -3012 & -1095 & 1950 & 42 \\
\hline $\mathrm{H}(25 \mathrm{~B})$ & -3926 & -445 & 2265 & 47 \\
\hline $\mathrm{H}(25 \mathrm{C})$ & -3600 & 449 & 2930 & 51 \\
\hline $\mathrm{H}(25 \mathrm{D})$ & -2331 & 757 & 3277 & 43 \\
\hline $\mathrm{H}(25 \mathrm{E})$ & -1397 & 114 & 2977 & 40 \\
\hline $\mathrm{H}(26 \mathrm{~B})$ & -1350 & -1287 & 3228 & 38 \\
\hline $\mathrm{H}(26 \mathrm{C})$ & -424 & -1487 & 3913 & 42 \\
\hline $\mathrm{H}(26 \mathrm{D})$ & 831 & -1391 & 3903 & 41 \\
\hline $\mathrm{H}(26 \mathrm{E})$ & 1179 & -1121 & 3183 & 43 \\
\hline $\mathrm{H}(26 \mathrm{~F})$ & 252 & -979 & 2474 & 39 \\
\hline $\mathrm{H}(27 \mathrm{~B})$ & -2228 & -2889 & 1062 & 35 \\
\hline $\mathrm{H}(27 \mathrm{C})$ & -2748 & -3706 & 359 & 38 \\
\hline $\mathrm{H}(27 \mathrm{D})$ & -1982 & -4164 & -156 & 52 \\
\hline $\mathrm{H}(27 \mathrm{E})$ & -703 & -3848 & 34 & 59 \\
\hline $\mathrm{H}(27 \mathrm{~F})$ & -186 & -3014 & 738 & 40 \\
\hline $\mathrm{H}(28 \mathrm{~A})$ & 624 & -1729 & 1283 & 39 \\
\hline $\mathrm{H}(28 \mathrm{~B})$ & 1849 & -2106 & 1441 & 46 \\
\hline $\mathrm{H}(28 \mathrm{C})$ & 2197 & -3026 & 1935 & 51 \\
\hline $\mathrm{H}(28 \mathrm{D})$ & 1295 & -3552 & 2284 & 59 \\
\hline $\mathrm{H}(28 \mathrm{E})$ & 72 & -3228 & 2101 & 48 \\
\hline
\end{tabular}




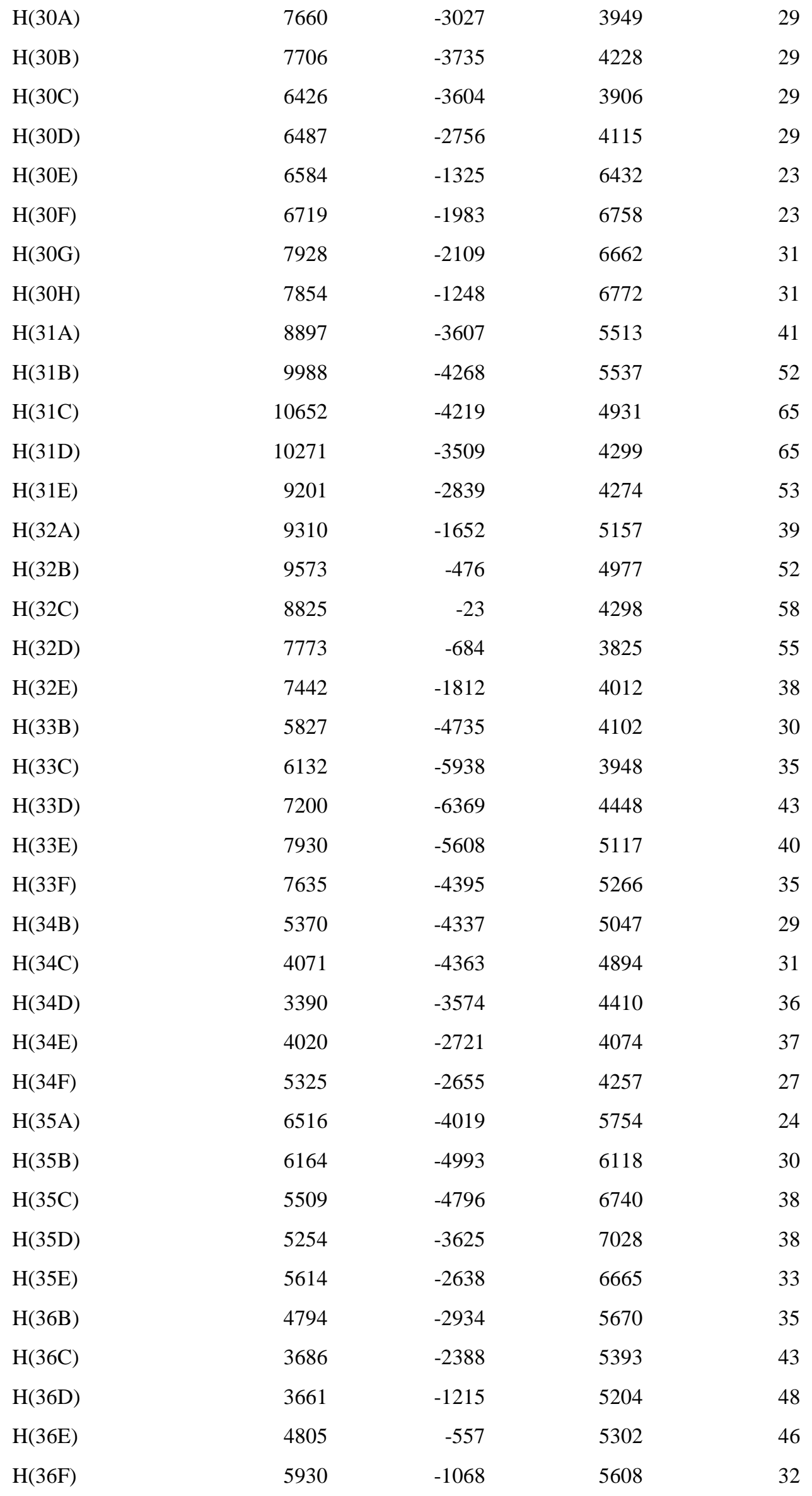




\begin{tabular}{|c|c|c|c|c|}
\hline $\mathrm{H}(37 \mathrm{~B})$ & 7732 & -716 & 5135 & 29 \\
\hline $\mathrm{H}(37 \mathrm{C})$ & 7562 & 489 & 5029 & 38 \\
\hline $\mathrm{H}(37 \mathrm{D})$ & 7248 & 1302 & 5658 & 39 \\
\hline $\mathrm{H}(37 \mathrm{E})$ & 7186 & 890 & 6404 & 34 \\
\hline $\mathrm{H}(37 \mathrm{~F})$ & 7398 & -330 & 6512 & 31 \\
\hline $\mathrm{H}(38 \mathrm{~A})$ & 8968 & -325 & 6125 & 30 \\
\hline $\mathrm{H}(38 \mathrm{~B})$ & 10279 & -211 & 6259 & 40 \\
\hline $\mathrm{H}(38 \mathrm{C})$ & 10965 & -1250 & 6235 & 45 \\
\hline $\mathrm{H}(38 \mathrm{D})$ & 10392 & -2385 & 6167 & 43 \\
\hline $\mathrm{H}(38 \mathrm{E})$ & 9087 & -2518 & 6035 & 35 \\
\hline $\mathrm{H}(1 \mathrm{SA})$ & 1190 & 8392 & 5023 & 121 \\
\hline $\mathrm{H}(1 \mathrm{SB})$ & 1059 & 7651 & 5239 & 121 \\
\hline $\mathrm{H}(2 \mathrm{SA})$ & 1333 & 4173 & 3424 & 113 \\
\hline $\mathrm{H}(2 \mathrm{SB})$ & 801 & 4693 & 3096 & 113 \\
\hline $\mathrm{H}(3 \mathrm{SA})$ & 8812 & 7156 & 8524 & 258 \\
\hline $\mathrm{H}(3 \mathrm{SB})$ & 8737 & 7389 & 9078 & 258 \\
\hline $\mathrm{H}(11 \mathrm{~F})$ & 3569 & 6936 & 2801 & 146 \\
\hline $\mathrm{H}(12 \mathrm{~F})$ & 2973 & 7937 & 3106 & 289 \\
\hline $\mathrm{H}(13 \mathrm{G})$ & 2036 & 7767 & 3533 & 189 \\
\hline $\mathrm{H}(14 \mathrm{G})$ & 1695 & 6597 & 3656 & 158 \\
\hline $\mathrm{H}(15 \mathrm{~F})$ & 2291 & 5596 & 3352 & 183 \\
\hline $\mathrm{H}(16 \mathrm{G})$ & 3229 & 5766 & 2924 & 179 \\
\hline $\mathrm{H}(4 \mathrm{SA})$ & 9473 & 4219 & 9405 & 163 \\
\hline $\mathrm{H}(4 \mathrm{SB})$ & 8792 & 3642 & 9311 & 163 \\
\hline $\mathrm{H}(21 \mathrm{~F})$ & 6322 & 238 & 10097 & 131 \\
\hline $\mathrm{H}(21 \mathrm{G})$ & 6388 & -533 & 9790 & 131 \\
\hline $\mathrm{H}(21 \mathrm{H})$ & 5615 & -121 & 9681 & 131 \\
\hline $\mathrm{H}(22 \mathrm{~F})$ & 6206 & 810 & 9365 & 193 \\
\hline $\mathrm{H}(22 \mathrm{G})$ & 6989 & 428 & 9491 & 193 \\
\hline $\mathrm{H}(23 \mathrm{G})$ & 6738 & 20 & 8693 & 183 \\
\hline $\mathrm{H}(23 \mathrm{H})$ & 6180 & -570 & 8817 & 183 \\
\hline $\mathrm{H}(24 \mathrm{G})$ & 5261 & 434 & 8720 & 205 \\
\hline $\mathrm{H}(24 \mathrm{H})$ & 5397 & -36 & 8222 & 205 \\
\hline $\mathrm{H}(25 \mathrm{~F})$ & 6534 & 780 & 8323 & 218 \\
\hline $\mathrm{H}(25 \mathrm{G})$ & 6208 & 1300 & 8720 & 218 \\
\hline $\mathrm{H}(26 \mathrm{G})$ & 5821 & 1685 & 7915 & 249 \\
\hline $\mathrm{H}(26 \mathrm{H})$ & 5100 & 1440 & 8100 & 249 \\
\hline $\mathrm{H}(26 \mathrm{I})$ & 5409 & 907 & 7704 & 249 \\
\hline
\end{tabular}




\begin{tabular}{lrrrr}
$\mathrm{H}(5 \mathrm{SA})$ & 6387 & -1554 & 10093 & 58 \\
$\mathrm{H}(5 \mathrm{SB})$ & 6447 & -861 & 9805 & 58 \\
$\mathrm{H}(31 \mathrm{~F})$ & 7887 & 4053 & 9676 & 187 \\
$\mathrm{H}(31 \mathrm{G})$ & 7724 & 3561 & 9149 & 187 \\
$\mathrm{H}(31 \mathrm{H})$ & 8122 & 3225 & 9640 & 187 \\
$\mathrm{H}(32 \mathrm{~F})$ & 6975 & 3338 & 9951 & 58 \\
$\mathrm{H}(32 \mathrm{G})$ & 6575 & 3748 & 9494 & 58 \\
$\mathrm{H}(33 \mathrm{G})$ & 6524 & 2277 & 9497 & 334 \\
$\mathrm{H}(33 \mathrm{H})$ & 7147 & 2359 & 9187 & 334 \\
$\mathrm{H}(34 \mathrm{G})$ & 6103 & 3173 & 8707 & 247 \\
$\mathrm{H}(34 \mathrm{H})$ & 5635 & 2535 & 8863 & 247 \\
$\mathrm{H}(35 \mathrm{~F})$ & 6694 & 2336 & 8221 & 152 \\
$\mathrm{H}(35 \mathrm{G})$ & 6575 & 1680 & 8526 & 152 \\
$\mathrm{H}(36 \mathrm{G})$ & 5694 & 1582 & 7716 & 173 \\
$\mathrm{H}(36 \mathrm{H})$ & 5333 & 2312 & 7903 & 173 \\
$\mathrm{H}(36 \mathrm{I})$ & 5274 & 1596 & 8160 & 173 \\
\hline
\end{tabular}

